\title{
Electric fields and electrical insulation
}

\author{
McAllister, lain Wilson
}

Published in:

IEEE Transactions on Dielectrics and Electrical Insulation

Link to article, DOI:

10.1109/TDEl.2002.1038656

Publication date:

2002

Document Version

Publisher's PDF, also known as Version of record

Link back to DTU Orbit

Citation (APA):

McAllister, I. W. (2002). Electric fields and electrical insulation. IEEE Transactions on Dielectrics and Electrical Insulation, 9(5), 672 - 696. https://doi.org/10.1109/TDEI.2002.1038656

\section{General rights}

Copyright and moral rights for the publications made accessible in the public portal are retained by the authors and/or other copyright owners and it is a condition of accessing publications that users recognise and abide by the legal requirements associated with these rights.

- Users may download and print one copy of any publication from the public portal for the purpose of private study or research.

- You may not further distribute the material or use it for any profit-making activity or commercial gain

- You may freely distribute the URL identifying the publication in the public portal

If you believe that this document breaches copyright please contact us providing details, and we will remove access to the work immediately and investigate your claim. 


\title{
Electric Fields and Electrical Insulation
}

\author{
I. W. McAllister \\ Department of Electric Power Engineering \\ Technical University of Denmark \\ Lyngby, Denmark
}

\begin{abstract}
The adoption of a field-theoretical approach to problems arising in the framework of electrical insulation is discussed with reference to six main topics, which have been addressed over the last 30 years. These include uniform field electrodes, Green's differential equation, electrode surface roughness, induced charge, electrostatic probes, and partial discharge transients, together with several follow-on aspects. Each topic is introduced and thereafter the progress achieved through the use of a field-theoretical approach is reviewed. Because the topics cover a wide spectrum of conditions, it is amply demonstrated that such an approach can lead to significant progress in many areas of electrical insulation.
\end{abstract}

\section{INTRODUCTION}

Over the last 25 to 30 years, the application of electric field theory to widely different aspects of electrical insulation has led to a more correct understanding of the underlying phenomena. Hence it is the purpose of the present paper to review briefly several important topics in which such progress has been achieved through adopting a field-theoretical approach.

Electric fields may be considered as the primary cause of insulation failure, or stated more cryptically: "no field, no problem". Thus it is clear that a detailed knowledge of the electric field associated with a system is a prerequisite to undertaking an assessment of the electrical insulation.

Since the advent of desk-top computing there has been an enormous increase in numerical field calculations. The technological developments in computer hardware have led to a corresponding increase in the complexity of the software available. The present review will not discuss computational aspects of electric fields, but rather concentrate on the situations in which a field-theoretical approach was adopted to study the problem under investigation. Such an approach has invariably led to a fuller understanding of the nature of the problem.

The basis for this conclusion is brought about by describing the progress achieved in six different topics. These encompass

1. uniform field electrodes and the significance of the inherent field non-uniformity;

2. use of Green's differential equation to assess electric field distributions:

3. characteristics of electrode surface roughness and the significance of the figure of merit for strongly electronegative gases and gas mixtures;
4. induced charge and how to quantify it;

5. electrostatic probes: their characteristics and evaluation of measurements; and

6. partial discharge transients: what is measured and its significance.

The first 3 topics essentially relate to the influence of electric fields on discharge onset conditions, while the last 3 discuss induced charge and its significance. It should be emphasized that the above topics are not reviewed in depth, although adequate references are provided in the text. This review focuses on the progress achieved through the application of a field-theoretical approach.

The past 30 years have seen many developments in the scientific world, not the least of which has been the introduction of the SI system of units. This has led to several changes of units, particularly with respect to gas pressure, e.g. from $\mathrm{mm} \mathrm{Hg} \rightarrow$ Torr $\rightarrow$ atm $\rightarrow$ bar $\rightarrow \mathrm{Pa}$ Several of these and others appear in the original Figures reproduced in this paper. Consequently, the relevant conversion factors are given in the Appendix.

\section{UNIFORM FIELD ELECTRODES}

\subsection{INTRODUCTION}

With respect to the fundamental macroscopic properties of gaseous dielectrics, there are several basic experiments, e.g. Paschen curve measurements and ionization current growth measurements, which employ uniform-field electrodes. Such an electrode consists essentially of a plane center section and a profiled edge section of decreasing radius of curvature. There are several well-known edge profiles such as Rogowski and Bruce, the details of which may be found in the literature.

Electrodes of the above type are so-named because it is inherently assumed that these generate a uniform electric field. With reference to 
such a field, it is necessary to begin with some clarification. Electrostatic fields frequently are considered to fall into two categories, viz. either uniform or non-uniform. As this classification imparts a very misleading impression of the nature of electrostatic field distributions, some comments on this aspect are required.

With respect to rectangular coordinates $x, y, z$, consider a uniform electric field of infinite extent oriented parallel to the $z$ axis. The corresponding equipotential surfaces are infinite planes parallel to the $x y$ plane. Hence by replacing two of these surfaces with conducting planes, a uniform field of finite extent in the $z$ direction can be realized mathematically.

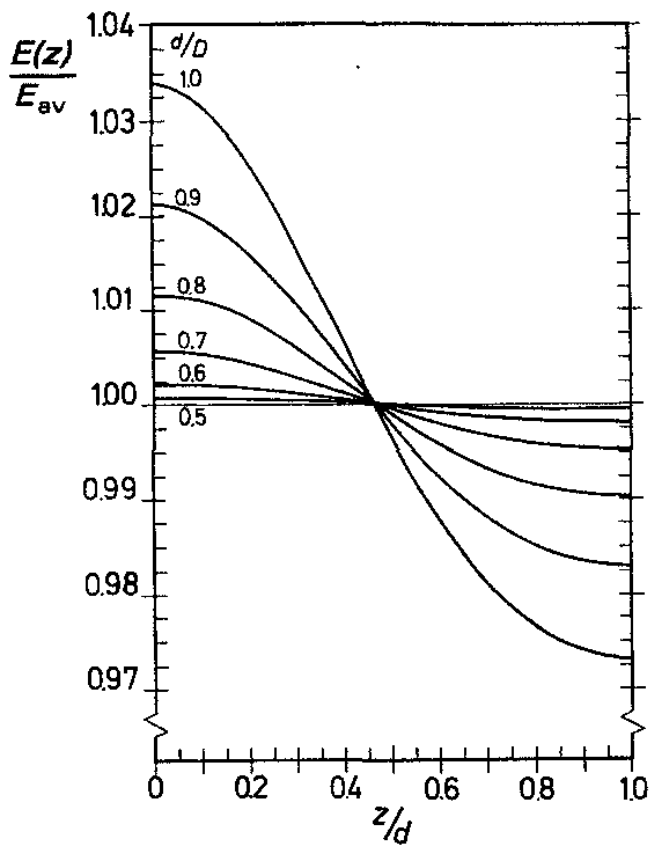

Figure 1. Axial field distribution of a uniform field gap. $d$ is the gap length, $D$ the overall flat diameter.

The related potential distribution is, of course, a solution of Laplace's equation. Consequently owing to the uniqueness theorem, see Kellogg [1], the infinite plane gap is the only electrode geometry to establish this potential distribution. In practice, all conductor geometries are of finite dimensions, and thus it is physically impossible to produce a uniform electric field; i.e. this field distribution belongs to the abstract world of mathematics, and should be considered a limiting condition.

In addition to the field non-uniformity introduced by the finite dimensions of the electrodes, there is also a field asymmetry which is a consequence of the normal asymmetric potential distribution: i.e. one of the electrodes is at potential $U$, while the other is at zero potential. This asymmetry leads to the axial field strength being higher at the HV electrode than at the grounded one. An example of the axial field distribution associated with uniform field electrodes is illustrated in Figure 1. If $d$ represents the gap spacing and $D$ is the overall flat diameter, then from this Figure it is clear that only for $d / D<0.5$ is the field non-uniformity $<0.1 \%$.
Another factor which affects the field non-uniformity is the presence of nearby grounded objects, e.g. the walls of the enclosing pressure/vacuum vessel. Because such objects will affect the edge field of the uniform field gap more than the axial field, this influence will become significant when using the electrodes at larger spacings, e.g. the maximum field strength in the edge region can readily exceed by several percent the maximum axial field strength, see [2].

\subsection{INFLUENCE OF INHERENT FIELD NON-UNIFORMITY}

In the early 1970s, Pedersen and colleagues [2-5] examined the effects of the inherent field non-uniformity of uniform field gaps with respect to $\mathrm{SF}_{6}$. Three aspects were investigated, viz. ionization current growth measurements [2,3], Paschen curve measurements [3], and edge breakdown $[4,5]$.

\subsubsection{IONIZATION CURRENT GROWTH $\mathrm{IN} \mathrm{SF}_{6}$}

Measurement of the steady-state ionization current growth is undertaken to provide data on the primary ionization coefficient $\alpha$, the secondary ionization coefficient $\gamma$, the attachment coefficient $\eta$, and the effective ionization coefficient $\bar{\alpha}=\alpha-\eta$. Traditionally these data are obtained via an analysis of the $\log (I), d$ plots, where $I$ is the gap current and $d$ the gap spacing, and also the main experimental variable. For finite values of $\gamma$, such plots exhibit an up-curving at high values of $d$.

In [2] however, for $\gamma=0$, it is shown that, for large values of $d$, the plots displayed either up-curving when the HV electrode is the cathode, or down-curving when the HV electrode was the anode, i.e. the influence of field asymmetry is evident. This feature is clearly illustrated in Figure 2. Experimental evidence of such behavior was subsequently provided by Boyd and Crichton, see Figure 3 and the Discussion in [2].

Consequently, the effect of the inherent field non-uniformity is to lead to appreciable errors in the derived $\gamma$ values. In addition, because upcurving is usually taken as evidence in support of the Townsend mechanism of breakdown, the effect of field non-uniformity upon ionization growth can lead to misinterpretation concerning the actual breakdown mechanism; i.e. Townsend contra streamer mechanism.

It is concluded that valid data for ionization coefficients in $\mathrm{SF}_{6}$, and other strongly electronegative gases, can be obtained when the field non-uniformity near the gap axis is $<0.1 \%[2,3]$. The expression 'valid data' implies that the experimental data can be analyzed as though these were uniform field data.

The field non-uniformity requirement was upheld in the ionization current measurements of Boyd and Crichton [6]. These indicated that, for the ranges $0.0067<p /$ bar $<0.53$ and $8.6<(E / p) / \mathrm{kV}(\mathrm{mm} \mathrm{bar})^{-1}<15$, the values of $\bar{\alpha} / p$ are a linear function of $E / p$, where $p$ denotes the gas pressure referred to $20^{\circ} \mathrm{C}$. This relation may be expressed as

$$
\bar{\alpha}(E, p)=\bar{\beta} E-\bar{\kappa} p
$$

From the measurements of Boyd and Crichton [6], identical to the data published in Table 5.6 of Kuffel and Zaengl $[7,8]$, the following values are obtained:

$$
\begin{array}{ll}
\bar{\beta}=27.7 \quad(\mathrm{kV})^{-1} \\
\bar{\kappa}=245 \quad(\text { bar mm })^{-1}
\end{array}
$$




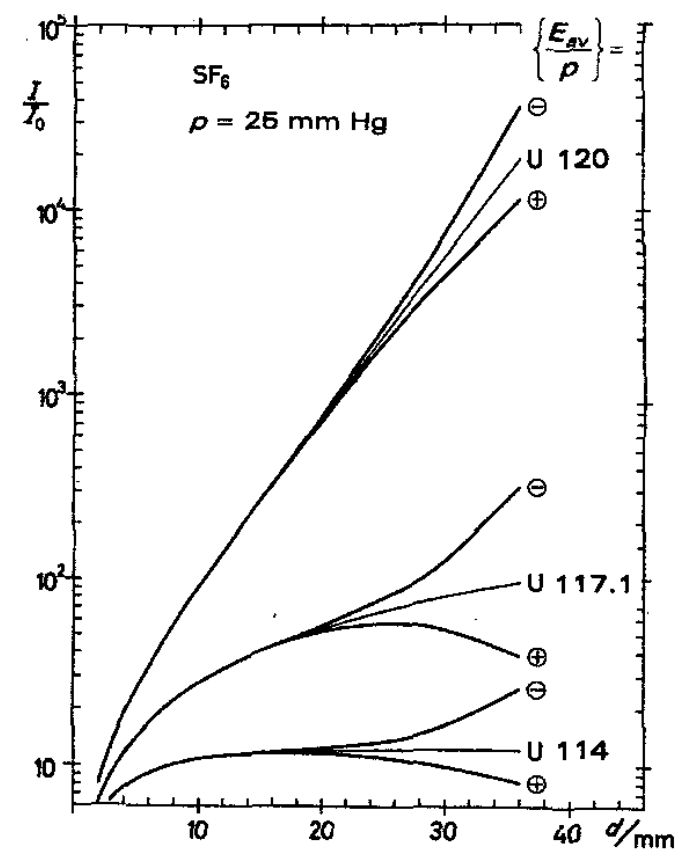

Figure 2. Theoretical $\log (I), d$ plots for both polarities and $\gamma=0$ [2]. Curves marked $U$ represent current growth in a strictly uniform field. The unit of $E_{\mathrm{av}} / p$ is $\left(\mathrm{V} \mathrm{cm}^{-1}\right) / \mathrm{mm} \mathrm{Hg}$.

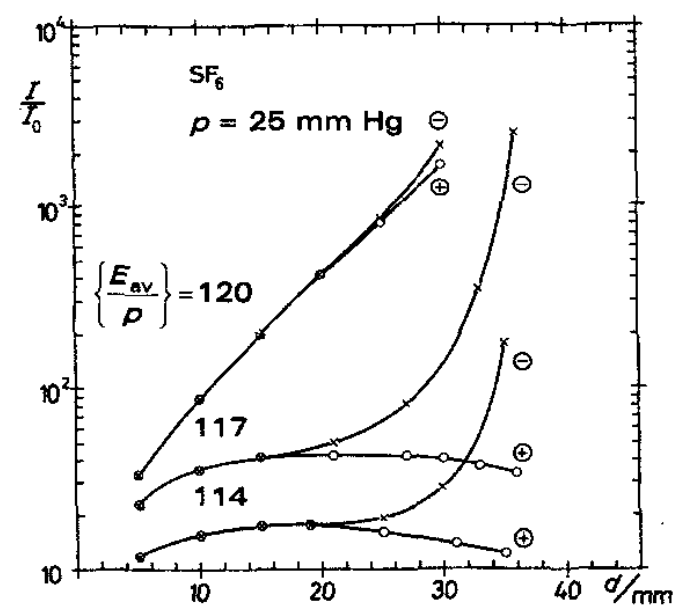

Figure 3. Experimental $\log (I), d$ plots for the same $E_{\text {av }} / p$ values [2].

The value of $E / p$ for which $\bar{\alpha}=0$ is called the limiting value. Thus from (1) we have for $\bar{\alpha}=0$

$$
\left(\frac{E}{p}\right)_{\mathrm{lim}}=\frac{\bar{\kappa}}{\overline{\bar{\beta}}}=8.83 \mathrm{kV}(\mathrm{mm} \mathrm{bar})^{-1}
$$

For $E / p$ values less than this value, $\bar{\alpha}$ is negative, which implies that all free electrons would be trapped eventually as negative ions.

Even with a degree of non-uniformity of $<0.1 \%$, it appears that a gas pressure of $\sim 0.1 \mathrm{MPa}$ will be the ultimate upper limit for an experimental determination of uniform field ionization data for $\mathrm{SF}_{6}[3,6]$.

\subsubsection{PASCHEN CURVE FOR $\mathrm{SF}_{6}$}

Paschen curve measurements refer to breakdown voltage measurements in a uniform field as a function of the product $p d$, where $p$ denotes the gas pressure, and $d$ the gap length. When expressed in this manner, it is inherently assumed that the ideal gas law is obeyed. Such measurements are an indication of the basic insulation strength of the gas.

In [3] Karlsson and Pedersen showed that, within the $E / p$ range of practical interest, valid Paschen curve data could be obtained so long as the initial ionization growth extends across the entire gap of length $d$. This implies that for a uniform field gap exhibiting a monotonically varying axial field distribution, the minimum axial field strength $E_{\min } \geqslant E_{\mathrm{lim}}$. For such a field distribution, the degree of field nonuniformity $\Delta E$ is defined as

$$
\Delta E \equiv \frac{E_{\mathrm{av}}-E_{\mathrm{min}}}{E_{\mathrm{av}}}
$$

where $E_{\text {av }}$ is the average field strength along the gap axis. If $U$ represents the applied voltage, then

$$
E_{\text {av }}=\frac{U}{d}
$$

The above $E_{\text {lim }}$ condition leads to the following relation [3]

$$
\Delta E \leqslant \frac{0.043}{p d /(\text { bar mm })}
$$

Such a condition was observed in the Paschen curve measurements of Boyd and Crichton [9]. These measurements indicated that, for the range $0.6<p d /($ bar $\mathrm{mm})<8$, the breakdown voltage $U_{\mathrm{s}}$ was a linear function of the product $p d$. This could be expressed in the form

with

$$
U_{\mathrm{s}}=U_{0}+\left(\frac{E}{p}\right)_{\mathrm{lim}} p d
$$

$$
\begin{array}{rlrl}
U_{0} & =0.38 & \mathrm{kV} \\
\left(\frac{E}{p}\right)_{\mathrm{lim}} & =8.84 & \mathrm{kV} /(\mathrm{mm} \text { bar })
\end{array}
$$

This value of $(E / p)_{\text {lim }}$ is in good agreement with that determined via the $\vec{\alpha}$ measurements. Further discussion of the $U_{0}$ and $(E / p)_{1 \text { im }}$ values can be found in Section 2.4.

From the analysis in [2,3], Karlsson and Pedersen concluded that uniform field breakdown voltage measurements are subjected to less severe limitations than the associated current growth measurements.

In 1972, the CIGRE Working Group on Insulating Gases, WG 15-03, considered field non-uniformity to be the ratio of the maximum (axial) field strength $E_{\text {rnax }}$ to the average field strength $E_{\text {av }}$ [10]. Thereafter, with respect to $\mathrm{SF}_{6}$, it was decided to accept breakdown data obtained for $E_{\max } / E_{\mathrm{av}} \leqslant 1.05$ as valid Paschen curve data. This value of nonuniformity implies that such data must be restricted to values of $p d<$ 1 bar mm. However, an examination of the relevant part of Table D3 of the CIGRE approved Paschen curve for $\mathrm{SF}_{6}$ indicates that the data in question do not in fact relate to valid Paschen curve data [11]. This is readily confirmed with the observation that, for large values of $d$, e.g. $d>10 \mathrm{~mm}$, the values of $E / p_{25}$ associated with breakdown are $<\left(E / p_{25}\right)_{l i m}$. It should be noted that CIGRE selected $25^{\circ} \mathrm{C}$ as the reference gas temperature. 
A linear regression analysis of the breakdown voltage data listed in Table D2 of the said CIGRE publication gave the following results: for $0.1 \leqslant p_{25} d /($ bar $m m) \leqslant 10, U_{0}=0.64 \mathrm{kV}$ and $\left(E / p_{25}\right)_{\lim }=$ $8.79 \mathrm{kV} /(\mathrm{num}$ bar) with a coefficient of determination of 0.99994 . If the analyzed $p d$ range is limited to values $<1$ bar mm (see above), there is effectively no change in the derived value of $\left(E / p_{25}\right)_{\text {limm }}$.

A comparison of the Boyd and Crichton values with those derived from the CIGRE approved data indicates a difference of $70 \%$ in the $U_{0}$ values but only $\sim 1 \%$ in the $\left(E / p_{25}\right)_{\text {lim }}$ values. This suggests that the absolute accuracies of the voltage measurements between the CIGRE data and those of Boyd and Crichton [9] differ considerably. Further discussion of Paschen curves is to be found in Section 2.4.

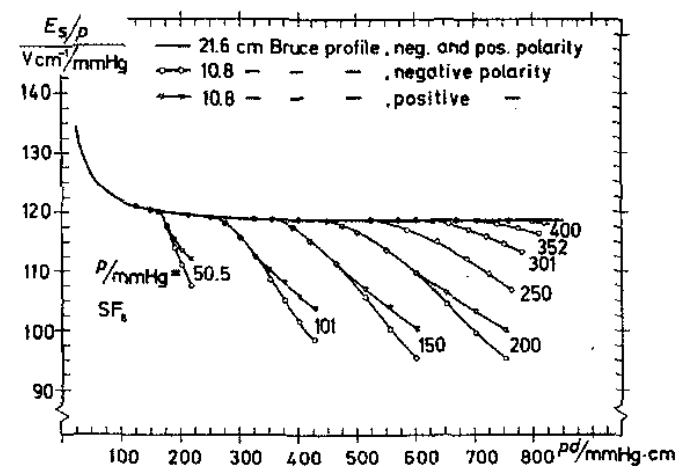

Figure 4. Edge (anomalous) breakdown in a uniform field gap in $\mathrm{SF}_{6}$ [5].

\subsubsection{EDGE BREAKDOWN}

During the 1960 s and early 1970 s, a new phenomenon was observed when undertaking breakdown voltage measurements in the strongly electronegative gases $\mathrm{SF}_{6}$ and $\mathrm{CCl}_{2} \mathrm{~F}_{2}$. It was discovered that the spark breakdown channels were occurring around the edges of the uniform field gap even at small gap spacings, e.g. $d=20 \mathrm{~mm}$ [5]. These anomalous breakdowns were known to occur at uniform field strengths

$$
E_{\mathrm{s}}=\frac{U_{\mathrm{s}}}{d}
$$

less than the limiting field strength $E_{\mathrm{lim}} . U_{\mathrm{s}}$ is the breakdown voltage. This behavior is illustrated in Figure 4. No satisfactory quantitative explanation was advanced for this edge (anomalous) breakdown phenomenon.

By considering ionization growth along the field line associated with the maximum edge field, it was possible to show that breakdown could occur if the growth was sufficient, see [5] for details. This critical condition was found to be fulfilled near the HV electrode, along a small fraction of the relevant field line. Thus the normal situation of discharge onset in a non-uniform field had become dominant, see Section 3.2 .

\subsection{EVALUATION OF STREAMER PARAMETER $K$}

A method of evaluating the streamer parameter $K$ for a particular gas can be illustrated with reference to $\mathrm{SF}_{6}$. A brief description of the streamer criterion is given in the Appendix.
The value of $K$ may be determined by applying the streamer criterion to a known critical condition; e.g. spark breakdown in a uniform field for which the value of the critical avalanche length $z_{0}$ can be defined precisely, viz. the gap length $d$ [12]. To ensure that $z_{0}=d$, the value of $E / p$ along the gap axis must nowhere be $<(E / p)_{\text {lim. }}$.

Applying the criterion to a strictly uniform field gives

$$
\bar{\alpha}_{\mathrm{s}} d=K
$$

$\bar{\alpha}_{s}$ is the value obtained from (1) for the measured Paschen curve breakdown field strength $E_{\mathrm{s}}$. Utilizing (1) and (10) we obtain

$$
U_{\mathrm{s}}=E_{\mathrm{s}} d=\frac{K}{\bar{\beta}}+\frac{\bar{\kappa}}{\bar{\beta}} p d
$$

where $U_{\mathrm{s}}$ is the Paschen curve breakdown voltage. Introducing $U_{0}=$ $K / \bar{\beta}$, and using (3) enables (11) to be written as

$$
U_{\mathrm{s}}=U_{0}+\left(\frac{E}{p}\right)_{\lim } p d
$$

The streamer criterion thus leads to a linear Paschen curve. The Paschen curve as a whole is of course nonlinear and exhibits a minimum value. However it is found experimentally that, over the range of $p d$ of interest to most practical applications, the curve is essentially linear.

Values of $\bar{\beta}$ and $U_{0}$ are derived from the precision measurements of Boyd and Crichton $[6,9]$, see (2) and (8). Thus for $K$ we have

$$
K=\bar{\beta} U_{0}=10.5
$$

When the data utilized in this evaluation reflect specific properties of the gas in question, there is no inherent reason to suppose that the value of $K$ for $\mathrm{SF}_{6}$ is pertinent to other strongly electronegative gases or gas mixtures. Moreover, for a weakly or non-attaching gas, $K$ need not have a constant value, but could be some function of $p z_{0}$.

It is important to emphasize that this method of evaluating $K$ is dependent upon the existence of valid experimental data for the functions $\bar{\alpha}(E, p)$ and $U_{\mathrm{s}}(p d)$. Because in practice it is impossible to produce a uniform electric field, it is necessary to assess the effect of the inherent field non-uniformity upon the measurements of $\bar{\alpha}$ and $U_{\mathrm{s}}$ and determine the maximum degree of non-uniformity which can be tolerated without affecting the validity of these experimental data. With reference to $\mathrm{SF}_{6}$ these restrictions were observed in the work of Boyd and Crichton [6,9], and consequently a valid evaluation of $K$ can be made utilizing their data.

The importance of the restrictions upon the degree of tolerable field non-uniformity becomes apparent if the CIGRE approved Paschen curve for $\mathrm{SF}_{6}$ is used to determine $K$. With the utilization of the CIGRE data negative values of $K$ can be derived.

With respect to the other major insulating gas, atmospheric air, the situation is complicated by a lack of proper $\bar{\alpha}$ data. Although there exists much data for dry air, there are essentially no data for humid air at atmospheric pressure. This lack may, to some extent, be circumvented by using an indirect approach: i.e. applying the streamer criterion under uniform field conditions and using the experimentally derived Paschen curve for atmospheric air, viz.

$$
\frac{E_{\mathrm{s}}}{p}=B+\frac{C}{\sqrt{p d}}
$$

where $B$ and $C$ are constants. This procedure leads to a quadratic dependence of $\bar{\alpha} / p$ upon $E / p$, see $[13,14]$ for details. There is some 
Table 1. Measured breakdown voltages in $\mathrm{SF}_{6} . d=16.000 \mathrm{~mm}$, $p_{20}=0.4946$ bar, $U_{s}=70.331 \mathrm{kV}, s(U)=3 \mathrm{~V}$.

\begin{tabular}{|r|l|}
\hline \hline Shot & $U, \mathrm{kV}$ \\
\hline 1 & 70.335 \\
2 & 70.333 \\
3 & 70.327 \\
4 & 70.333 \\
5 & 70.332 \\
6 & 70.329 \\
7 & 70.334 \\
8 & 70.327 \\
9 & 70.330 \\
10 & 70.333 \\
\hline
\end{tabular}

evidence to support this relation. However, because a quadratic relation always implies that $\bar{\alpha} \geqslant 0$, the disadvantage of such an $\bar{\alpha}$ function is that its usefulness is restricted to monotonic field distributions.

\subsection{PASCHEN CURVES FOR STRONGLY ELECTRONEGATIVE GASES AND GAS MIXTURES}

In practice, the $E / p$ range of interest for any strongly electronegative gas or gas mixture will be $\sim(E / p)_{\text {limn }}$. In this range of $E / p$, the magnitude of $\bar{\alpha} / p$ is the difference between much larger, approximately equal quantities which vary rapidly with $E / p$; viz. those quantities representing the electron production $(\alpha)$ and attachment $(\eta)$ processes.

As a first approximation, the variation of $\bar{\alpha} / p$ with $E / p$ can be considered to be linear. Consequently, on the basis of this assumption and the use of the streamer criterion, the Paschen curve breakdown voltage $U_{\mathrm{s}}$ can for a specific $p d$ range be represented by a linear expression, viz. that given by (12).

With the introduction of the figure of merit for a strongly electronegative gas or gas mixture in 1980, see Section 4.2, there arose a requirement to undertake Paschen curve measurements on such gases. This prompted the construction of new high precision measuring systems; e.g. see [15]. As a control, the system in [15] was used initially to undertake Paschen curve measurements on $\mathrm{SF}_{6}$.

The measured breakdown voltage was found to be an exceptionally well defined quantity, and the results were repeatable with an extreme degree of accuracy. To illustrate the accuracy which can be obtained, Table 1 shows a typical sequence of measurements for $\mathrm{SF}_{6}$. The average value of the measured voltages is $U_{\mathrm{s}}=70.331 \mathrm{kV}$ with a standard deviation of $3 \mathrm{~V}$.

The variation of the measured $U_{\mathrm{s}}$ values with $p d$ fitted very closely to a straight line. The values of $U_{0}$ and $(E / p)_{\text {lim }}$ in (12) could thus be found by linear regression. The coefficient of determination was 0.999998 . The results obtained were

$$
\begin{aligned}
U_{0} & =0.35 \mathrm{kV} \\
\left(\frac{E}{p}\right)_{\lim } & =8.86 \mathrm{kV} /(\mathrm{mm} \text { bar })
\end{aligned}
$$

These results confirm the validity of the original values, see (8), reported by Boyd and Crichton [9].
This linear dependence has been verified for a very large number of strongly electronegative gases and gas mixtures, see [16-21]. With one exception $\left(\mathrm{c}-\mathrm{C}_{4} \mathrm{~F}_{8}\right)$, the coefficient of determination was, over the greatest $p d$ range for each gas/gas mixture, not less than 0.99999 .

However, in itself a linear Paschen curve is not sufficient. It is necessary to check that the derived $U_{0}$ and $(E / p)_{\text {lim }}$ data fulfill the field non-uniformity requirement.

This necessary field control was not exercised by Berger and Senouci [22], who undertook Paschen curve measurements in $\mathrm{SF}_{6}$ with a point/ plane gap, viz. a hemispherically-ended rod of tip radius $8 \mathrm{~mm}$ with a gap spacing of $5 \mathrm{~mm}$. Upon applying a linear regression fit, i.e.

$$
U_{\mathrm{s}}=A+B p d
$$

to the breakdown data quoted in Table 1 of Berger and Senouci, gave the following values [23]

$$
\begin{array}{ll}
A=3.74 & \mathrm{kV} \\
B=6.96 & \mathrm{kV} /(\mathrm{mm} \text { bar })
\end{array}
$$

with a coefficient of determination of 0.9997 . Consequently, although a certain degree of linearity is displayed, the discrepancy between the Paschen parameters, see (15), and those derived from the Berger and Senouci measurements, is considerable. This feature emphasizes clearly the importance of adequate field control.

Furthermore, in [23] it was indicated that the necessary condition to obtain valid Paschen curve data, i.e. $z_{0} / d=1$, is met for $p<$ 0.036 bar, a value which lies outside the pressure range considered in [22], viz. $0.25 \leqslant p /$ bar $\leqslant 4$. Consequently it is evident that the breakdown measurements reported by Berger and Senouci do not pertain to those associated with the Paschen curve for $\mathrm{SF}_{6}$.

Although a moderately non-uniform field was produced by the test gap adopted by Berger and Senouci, see [23], an apparently linear breakdown voltage curve could be obtained experimentally. Curves of this type were denoted subsequently as 'illusory Paschen' curves; see [24].

For a moderately non-uniform field, the characteristics of such curves have been deduced from an analytical linear regression analysis of the theoretical breakdown voltage relationship. Full details are given in [24]. The analysis indicates that the gradient of the theoretical $U_{s}$ curve is given approximately by $\eta_{\mathcal{S}}(E / p)_{\text {lim }}$, where $\eta_{\mathcal{S}}$ is the field utilization factor of the test gap, defined as

$$
\eta_{S} \equiv \frac{E_{\mathrm{av}}}{E_{\max }}
$$

Finally, it should be added that $\mathrm{SF}_{6}[25]$ and other strongly electronegative gases are non-ideal gases. For $\mathrm{SF}_{6}$, this behavior should be taken into account for $p>1$ bar. However the measurements discussed in this Section were all made at $p<1$ bar.

\subsection{DISCUSSION}

From a quantitative knowledge of the field distribution associated with a uniform field gap, it was possible to analyze and explain different aspects of the related discharge behavior of $\mathrm{SF}_{6}$. The analysis enabled the limits to the use of uniform field electrodes to be delineated. 
The limit relating to Paschen curve measurements is of particular significance, because this condition allows such breakdown voltage measurements to be assessed quantitatively. Through an assessment of several sets of published data, the need to control all such measurements is clearly demonstrated, if on the basis of these, general quantitative interpretations are to be put forward.

An empirical approach to the evaluation of the streamer parameter $K$ for the gas in question is presented. In essence such an approach is dependent on the availability of uniform field data for $\bar{\alpha}(E, p)$ and $U_{s}(p d)$ obtained under the strict field conditions outlined in the preceding Sections.

\section{GREEN'S DIFFERENTIAL EQUATION}

\subsection{THE EQUATION AND ITS USE}

With the development of the charge simulation method of field calculation at the Technical University of Munich in the late 1960s and early 1970s [26-33], a neglected equation linking the differential geometry of equipotential surfaces with the field parameters was resurrected. Initially this equation was considered to have originated with the spread of vector analysis at the beginning of the twentieth century, but subsequently it was traced to Green's Essay on potential theory from 1828, see [34] for details.

For a Laplacian field, the field strength $E$ at a given point $P$ in space and the mean curvature $H$ of the equipotential surface through $P$ are related by the ordinary differential equation

$$
\frac{d E(z)}{d z}+2 H(z) E(z)=0
$$

where $z$ is a coordinate along the line of force through P. For regular surfaces, $H$ is defined as

$$
H=\frac{1}{2}\left(\frac{1}{\rho_{1}}+\frac{1}{\rho_{2}}\right)
$$

where $\rho_{1}$ and $\rho_{2}$ are the principal radii of curvature of the equipotential surface at $\mathrm{P}$.

With its retrieval, Green's equation was used to assess numerical field calculations, e.g. see $[26,33]$. Such a situation arose because a knowledge of the inherent conductor geometries is a prerequisite of any numerical method of solving Laplace's equation. The determination of the mean curvature of the different conductors is thus completely independent of the field calculations. Consequently, the fulfillment of Green's equation at a conductor surface can be used as a check on the accuracy of the field calculations. For example, if the conductor as a whole is considered to be associated with $z=0$, then for the selected location we compare the value obtained for

$$
-\frac{1}{E(0)}\left(\frac{d E(z)}{d z}\right)_{z=0}
$$

with the corresponding value of $2 H(0)$.

Green's equation can also provide insight into how the conductor geometry influences the local field distribution. For example, if we are primarily interested in the field along a line of force in the proximity of the surface of a $\mathrm{HV}$ conductor, we may consider $H(z)$ to be constant. For small $z$, a first approximation to (19) will then be [34]

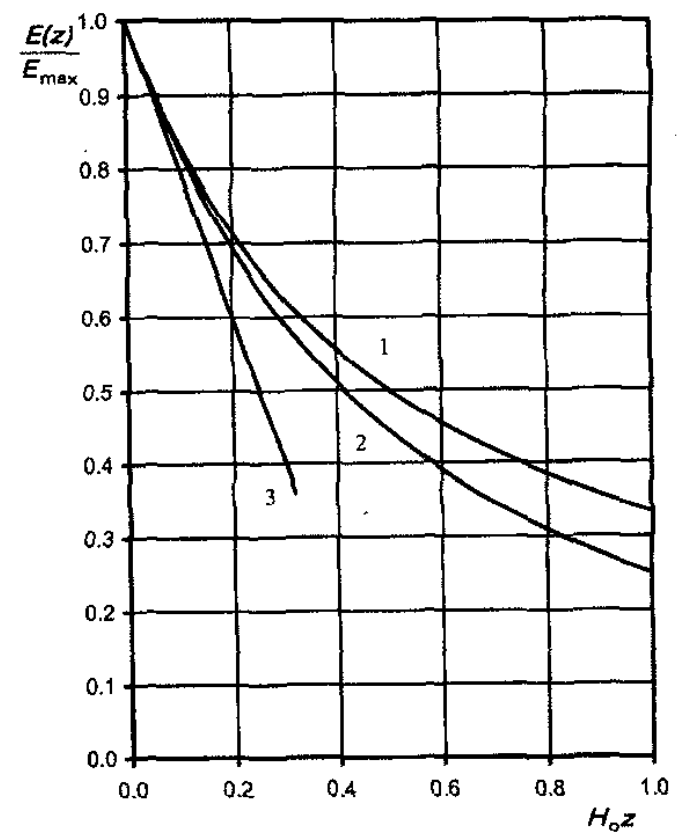

Figure 5. Electric field distributions. 1 isolated circular cylinder 2 isolated sphere; 3 approximation.

$$
E(z)=E(0)[1-2 H(0) z]
$$

in which $z$ is the distance along the field line in question from the electrode surface and $H(0)$ the mean curvature of the electrode surface at the location of the field line of interest.

The range of validity of (22) can be determined by examining the fields of two simple conductor geometries, viz. an isolated sphere and an isolated circular cylinder. These geometries exhibit a unique feature, viz. a constant mean curvature, and in addition these encompass the overall $\left(\rho_{1}, \rho_{2}\right)$ variation of interest, i.e.

(a) $\rho_{1}$ finite, $\rho_{2}$ finite and $\rho_{1}=\rho_{2}$;

(b) $\rho_{1}$ finite, $\rho_{2}$ infinite.

These thus delineate limiting situations for electrodes of finite dimensions.

The electric field distributions associated with these two conductor geometries are illustrated in Figure 5, together with the approximate distribution (22). Due to the high degree of symmetry associated with these geometries, $E(0)$ is in fact the maximum field strength $E_{\max }$ everywhere on the surface. However as we are invariably interested in the field line associated with $E_{\max }$, we will use this latter designation in future discussion. In addition, the value of the electrode mean curvature associated with $E_{\max }$ will be denoted as $H_{0}$.

From Figure 5, it is evident that, for $H_{0} z<0.1$, (22) represents a good approximation to the field distribution near a HV electrode. Thus on the basis of (22), it is possible to check the field distribution in the vicinity of a conductor surface. For example if, as in Figure 5 , the field distribution $E(z) / E_{\max }$ is plotted as a function of the normalized distance $H_{0} z$ along the field line, then the gradient of the resulting graph at $H_{0} z=0$ will be independent of both $H_{0}$ and $E_{\max }$ and will 


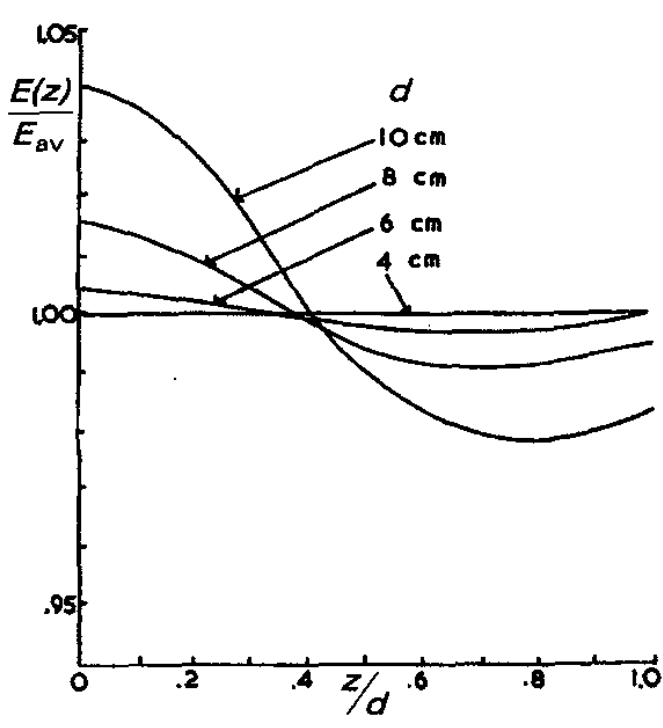

Figure 6. Axial electric field distribution for uniform field electrodes [35]. $E_{\text {ay }}$ is the average field strength.

have a value of -2 for convex surfaces. This characteristic gradient of -2 can be used as a checking factor.

The only major exception to the general rule is a plane surface for which $H(0)=0$. Hence in this case the gradient of the graph should be zero at the conductor surface. Other surfaces can also display $H(0)=0$. However, as this implies that $\rho_{1}=-\rho_{2}$, such locations are invariably associated with low field regions and are thus of little interest in electrical insulation studies. Such conditions will also apply to concave surfaces, which will yield a gradient of +2 .

The above procedure provides a simple method of controlling published computations of electric fields. This aspect is illustrated in Figure 6 which shows the computed axial field distribution between uniform field electrodes [35]. From an examination of this Figure it is obvious that, at the electrodes, the gradient of the field distribution curves is not zero. Hence the associated field calculations are in considerable error.

\subsection{SURFACE CURVATURE FACTOR $\zeta$}

The fact that the field distribution in the vicinity of an electrode is controlled by the electrode mean curvature alone enables a theoretical basis for the surface curvature factor $\zeta$ to be established [36]. This factor had been introduced on the basis of experimental measurements [37, 38].

For a non-uniform field, the breakdown or corona onset field strength $E_{0}$ for a gaseous dielectric will, for ideal (smooth) electrodes, be greater than the limiting value $\left(E_{\text {lim }}\right)$ associated with ionization growth. This feature can be taken into account by defining a surface curvature factor $\zeta$, viz.

$$
\frac{E_{0}}{p} \equiv \zeta\left(\frac{E}{p}\right)_{\lim } \quad \zeta>1
$$

where $p$ denotes gas pressure. $E_{0}$ represents the value of the maximum macroscopic field strength at the HV electrode.

Application of the streamer criterion, see Appendix, to the approximate field distribution allows an expression for $\zeta$ to be determined. However $\zeta$ is dependent on the gas in question. For $\mathrm{SF}_{6}$ and other strongly electronegative gases or gas-mixtures, a linear variation of the net ionization coefficient $\bar{\alpha}(E, p)$ is assumed within the $E / p$ range of practical interest, viz.

$$
\bar{\alpha}(E, p)=\bar{\beta} E-\bar{\kappa} p
$$

see also Section 2. Upon using the streamer criterion, we obtain the expression

$$
\zeta=1+\sqrt{\frac{4 H_{0} K}{p \bar{\kappa}}}
$$

Moreover, the corresponding critical avalanche length is given by

$$
H_{0} z_{0}=\sqrt{\frac{H_{0} K}{p \bar{\kappa}}}
$$

For $\mathrm{SF}_{6}$, an examination of this parameter indicates that for $p / H_{0}>5$ (bar $\mathrm{mm}$ ), $H_{0} z_{0}<0.1$, and thus the use of the approximate field distribution (22) is justified.

For a weakly electronegative gas such as atmospheric air, it is necessary to adopt a somewhat different approach. This situation arises because for such gases, $\bar{\alpha}$ is less sensitive to changes in $E$. Hence for the same electrode geometry, the critical avalanche length will be longer than that for a strongly electronegative gas. Consequently the validity of the approximate field distribution, (22), with respect to onset calculations is restricted. This limitation can be circumvented by first examining the variation of $\zeta$ for the two limiting field distributions, viz. those associated with the isolated spherical conductor and the isolated circular cylindrical conductor. The results of such calculations are shown in Figure 7. Full details can be found in [14]. These two curves can be considered to be the limiting curves for all electrode geometries which are associated with divergent fields. Moreover, for $p / H_{0}=1$ bar $\mathrm{mm}$, the difference between these curves is $<4 \%$, and hence in practice it is possible, via a regression analysis, to represent these by a single equation, see [14] and the discussion therein.

As a means of illustrating the difference between the onset conditions for $\mathrm{SF}_{6}$ and atmospheric air, it is of interest to examine the variation of the normalized critical avalanche length $H_{0} z_{0}$. This variation is shown in Figure 8. For atmospheric air it is seen that, for $p / H_{0}>$ 500 bar mm, $H_{0} z_{0}<0.1$, such that (22) becomes a valid approximation for the field distribution in the vicinity of the electrode. Whereas for $\mathrm{SF}_{6}$ the $H_{0} z_{0}$ condition is already fulfilled for $p / H_{0}>5$ bar mm.

\subsection{DISCUSSION}

Although Green's differential equation is widely used to assess the validity of numerical field calculations, the linking of the differential geometry of equipotential surfaces with the electric field distribution enables a deeper insight into how the electrode geometry controls the local field non-uniformity. Due to this behavior, the discharge onset field strength is controlled by the electrode mean curvature. 


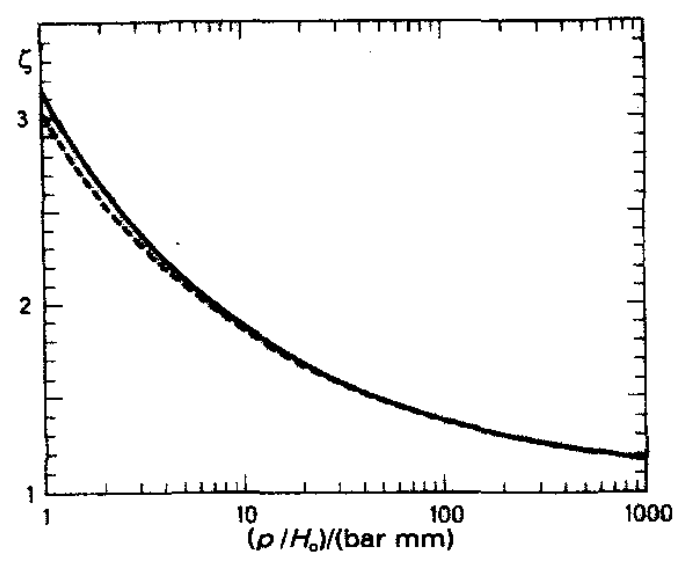

Figure 7. Surface curvature factor $\zeta$ for atmospheric air [14]. Full line: spherical field distribution. Dashed line: cylindrical field distribution.

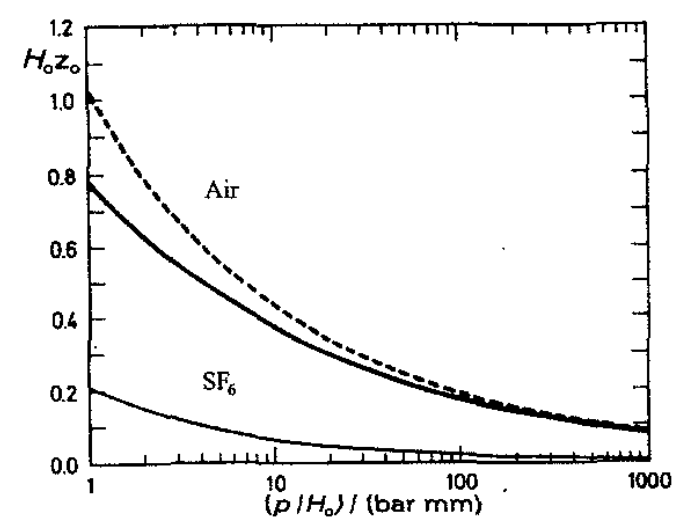

Figure 8. Normalized critical avalanche $H_{0} z_{0}$ for atmospheric air and $\mathrm{SF}_{6}$. Full line: air, spherical field distribution. Dashed line: air, cylindrical field distribution.

\section{ELECTRODE SURFACE ROUGHNESS}

\subsection{INTRODUCTION}

With the introduction of compressed $\mathrm{SF}_{6}$ as an insulating medium, it was discovered that, for gas pressures in excess of $0.1 \mathrm{MPa}$, the insulation strength of the system was less than that expected. Such a reduction may occur for several reasons, one of which can be attributed to the effect of the inherent surface roughness of the electrodes [39-42]. This roughness, which is a feature of the production processes employed in the manufacture of the conductors, leads to the existence of microscopic regions in which the field strength is higher than that associated with the ideal macroscopic electrode geometry: i.e. the surface roughness produces a perturbation of the macroscopic electric field.

Although it is possible to quantify the influence of electrode surface roughness upon insulation strength in terms of the measured breakdown voltages, it is difficult to do so directly in terms of the actual roughness geometry. This situation arises from the complex geometrical structure of an actual electrode surface. Nevertheless, an estimate and understanding of this influence can be obtained by considering simple models of a rough surface. A simple model may be defined as one for which the basic electrostatic parameters can be readily determined, viz the field enhancement factor $m$, and the field distribution associated with the model $E_{\max }$. The former parameter quantifies the increase in field strength due to the protrusion with respect to the field strength $E_{\mathrm{a}}$ at the associated (idealized) macroscopic electrode surface: i.e. the field enhancement factor $m$ is defined as

$$
m \equiv \frac{E_{\mathrm{max}}}{E_{\mathrm{a}}} \quad m \geqslant 1
$$

On this basis, many roughness models were considered during the 1970s; viz. hemispheres [43], portions of spheres [44-46], spheres [47, 48], prolate hemispheroids [49-51] and hemispherical tipped rods [48]. Together these cover a wide range of $m$ values. However, as all such geometries are rotationally symmetric, these exhibit similar field distributions. To cater for this electrostatic parameter, it is necessary to consider quite different protrusion geometries, i.e. those exhibiting a cylindrical symmetry, such as a hemicylinder [52], or a circular wire [53]. Although such protrusions display a smaller $m$ value than the corresponding rotational protrusions, the cylindrical protrusions have a less non-uniform field distribution. The overall field distribution is important when considering the growth of ionization. Other field characteristics can also be taken into account: e.g. the mutual effects of neighboring protrusions $[52,54]$, and the influence of the mean curvature $H_{\mathrm{a}}$ of the macroscopic electrode [55].

The roughness modeling approach was adopted initially by Agapov and Razevig in 1972 with respect to compressed air [49]. However, as interest was primarily focused on $\mathrm{SF}_{6}$ insulation, that study appears to have been generally overlooked, such that the major developments in modeling occurred subsequently with respect to $\mathrm{SF}_{6}$.

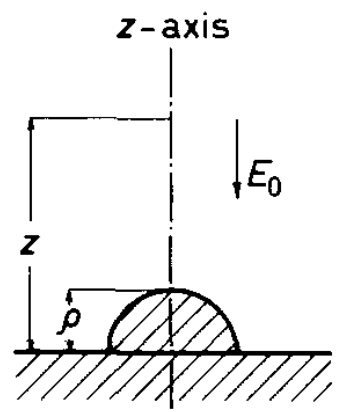

Figure 9. Simple model of a rough electrode surface.

\subsection{SURFACE ROUGHNESS FACTOR $\xi$}

This factor was introduced in 1974 by Mosch and Hauschild to account for the reduction observed in the measured breakdown voltage of $\mathrm{SF}_{6}$ below that predicted on the basis of the idealized macroscopic electrode geometry $[37,38]$. A theoretical basis for this factor was provided in 1975 by Pedersen, who applied the streamer criterion to the field 


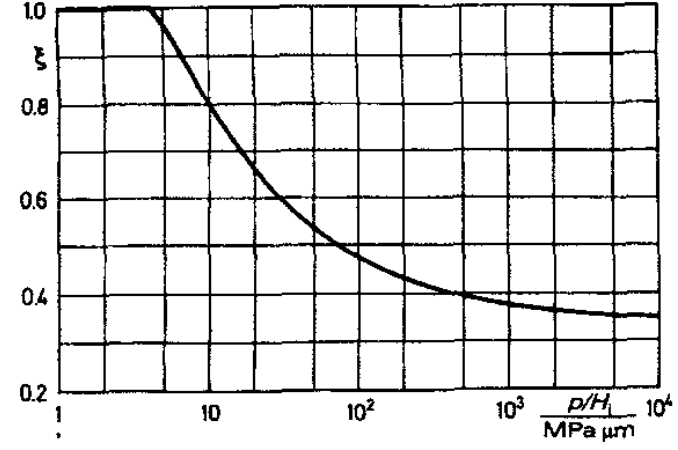

Figure 10. Surface roughness factor $\xi$ for $\mathrm{SF}_{6}[36]$.

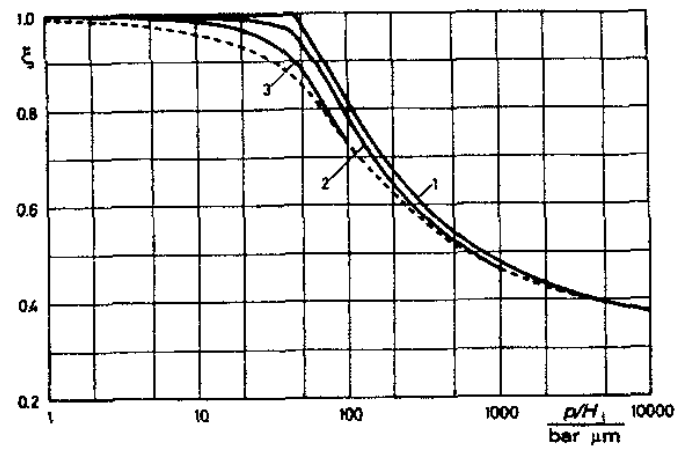

Figure 11. Surface roughness factor $\xi$ for $\mathrm{SF}_{6}$ [55]. $1: H_{\mathrm{a}} / p=0$

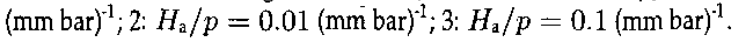
Dashed line: $r / R=0.01$ limit. For the intersecting sphere model, $r / R$ is the ratio of the sphere radii.

perturbation associated with a simple model $[43,56,36]$, see Figure 9. The roughness was represented by a protrusion in the form of a small conducting hemisphere of radius $\rho$ on a smooth planar electrode. This electrode implies that the macroscopic electric field is represented by a uniform field of infinite extent. From (25) it is clear that, as $H_{0} \rightarrow 0$, $\zeta \rightarrow 1$ and thus $\left(E_{0} / p\right)$ for the planar electrode is $(E / p)_{\mathrm{lim}}$. The surface roughness will however reduce the value of $\left(E_{0} / p\right)$, such that the surface roughness factor can, in this instance, be defined as

$$
\frac{E_{0}}{p} \equiv \xi\left(\frac{E}{p}\right)_{\mathrm{lim}} \quad 0<\xi<1
$$

By combining the field distribution associated with $E_{\max }$, the streamer criterion and (28), the onset condition for $\mathrm{SF}_{6}$ can be expressed in terms of the protrusion mean curvature $H_{\mathrm{i}}$ as, see $[43,56,36]$ for details,

$$
\frac{H_{\mathrm{i}}}{p}=\frac{\bar{\kappa}}{K}\left(1-\left(\frac{27}{4} \xi(1-\xi)^{2}\right)^{\frac{1}{3}}\right)
$$

In this instance $H_{\mathrm{i}}=1 / \rho$. The $\xi$ variation for $\mathrm{SF}_{6}$ is illustrated in Figure 10. It is seen that the surface roughness has no effect upon $\xi$ if

$$
\frac{p}{H_{\mathrm{i}}}<40 \quad \text { bar } \mu \mathrm{m}
$$

A similar type of behavior is displayed by other protrusion models, e.g. see $[52,53,55]$. 'The critical value of $p / H_{\mathrm{i}},\left(p / H_{\mathrm{i}}\right)_{\mathrm{C}}$, is a function of both $m$ and $E(z) / E_{\max }$.
Although it could appear that the value of $\left(p / H_{\mathrm{i}}\right)_{\mathrm{c}}$ must be of primary importance for design purposes, this parameter was later shown to be a feature of the models which represented the macroscopic electrode by a plane conductor $\left(H_{\mathrm{a}}=0\right)$, see [43-54]. This limitation is avoided with an intersecting sphere model for which $H_{\mathrm{a}}>0$ [55]. An intersecting cylinder model is another possibility. The variation of $\xi$ for the intersecting sphere model is shown in Figure 11, from which it is clear that there is now no critical $\left(p / H_{\mathrm{i}}\right)$ value when $H_{\mathrm{a}}>0$.

Nevertheless, the value of $\left(p / H_{\mathrm{i}}\right)_{\mathrm{c}}$ still retains a significance. From Figure 11 it is clear that, for $\left(p / H_{\mathrm{i}}\right)<\left(p / H_{\mathrm{i}}\right)_{c}$, the reduction in $\xi$ is small $(<10 \%)$, while, for $\left(p / H_{\mathrm{i}}\right)>\left(p / H_{\mathrm{i}}\right)_{\mathrm{c}}$, the reduction can be substantial ( $\gg 10 \%)$. In [55], it is shown that this transition in the $\xi$ values is associated with the electron avalanche development occurring either partially or wholly within the field perturbation.

With respect to multiple protrusions, the behavior of $\xi$ is again dependent on the two field parameters. Owing to the existence of other protrusions, these parameters are reduced in both magnitude and spatial extent, e.g. see [52]. Consequently, in comparison to the corresponding isolated protrusion, the associated $\left(p / H_{\mathrm{i}}\right)_{\mathrm{c}}$ will be greater, while the reduction in $\xi$ will be less.

\subsubsection{DEVIATIONS FROM THE PASCHEN LAW}

In the early 1970s, deviations from Paschen's law for high pressure $\mathrm{SF}_{6}$ were reported [57]. On the basis of the reported deviations, it was possible to provide an explanation in terms of the influence of surface roughness $[58,59]$. Such explanations usually are not discussed in terms of the surface roughness factor.

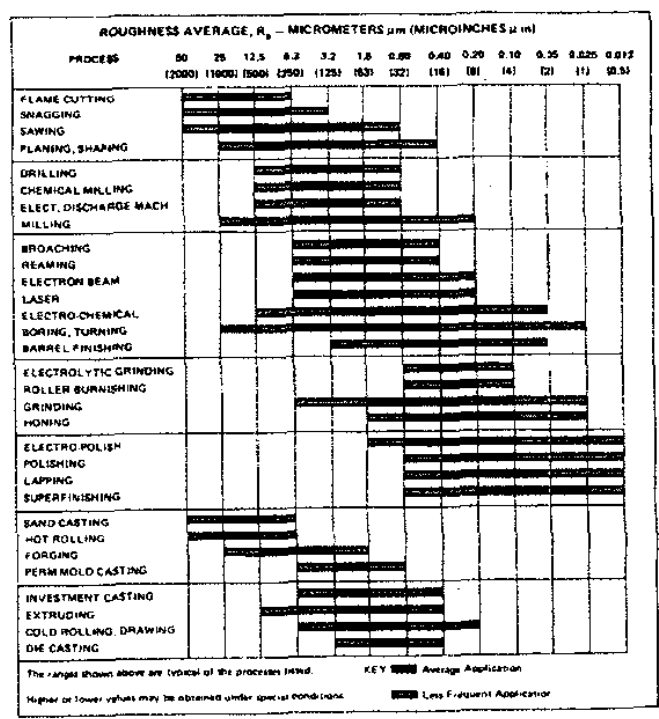

Figure 12. Surface roughness dimensions associated with different production processes $[53,65]$.

\subsubsection{MODEL ROUGHNESS EXPERIMENTS}

In addition to theoretical studies employing simple models, there were also many experimental studies of roughness effects. Such stud- 
ies were based on two different approaches: viz. (a) the use of geometrically well-defined artificial protrusions mounted on an electrode, and (b) the use of an electrode having a surface finish comparable with that related to production processes, but which in comparison to (a) is ill-defined. The latter approach represents, with respect to insulation strength, the normal operating situation and the results from any such investigation are directly applicable to a practical situation. The former approach using model protrusions, e.g. a hemisphere [60], spheres [48, $61,62]$, prolate hemispheroids [63], or a circular wire [64], provides a well-defined perturbation of the macroscopic electric field.

In the former approach, the dimensions of the protrusions employed were $\geqslant 100 \mu \mathrm{m}$. However the inherent roughness associated with a conductor surface is dependent upon the production process employed see Figure $12[53,65]$. This indicates that with the latter approach most surfaces are to be associated with roughness dimensions $<25 \mu \mathrm{m}$. It should be noted however that the roughness parameters defined in various standards are purely empirical, and thus the above value should only be taken as a coarse guide to the actual dimensions of the rough surface.

For $\mathrm{SF}_{6}$ at a gas pressure $p<5$ bar, the theoretical modeling indicates that the roughness dimensions associated with a small reduction in $\xi$ are $<100 \mu \mathrm{m}$. Hence it may be concluded that experimental studies which use model protrusions do not in fact deal with field perturbations, but rather with self-established macroscopic field distributions.

\subsection{FIGURE OF MERIT FOR A STRONGLY ELECTRONEGATIVE GAS/GAS MIXTURE}

From the analysis of the discharge onset condition of simple protrusion models, it is seen that this condition can always be expressed in the form

$$
\frac{p}{H_{\mathrm{i}}}=\frac{K}{\bar{\kappa}} f(\xi)
$$

The function $f(\xi)$ depends upon the field parameters of the model, while the ratio $K / \bar{\kappa}$ represents the practical dielectric properties of the gas. The existence of this common ratio led Pedersen to propose in 1980 that it could be used as a figure of merit for strongly electronegative gases, because its value would reflect the sensitivity of a gas to electrode surface roughness [66]; i.e. the smaller $K / \bar{\kappa}$ is, the more sensitive is the gas to the microscopic field perturbations produced by surface roughness. The figure of merit $M$ of a strongly electronegative gas, or gas mixture, is thus defined as

$$
M \equiv \frac{K}{\bar{\kappa}}
$$

A direct determination of $M$ from this definition would require two types of measurement to be undertaken in uniform field gaps, viz. the determination of $\bar{\alpha}(E, p)$ and measurement of the linear part of the Paschen curve $U_{\mathrm{s}}(p d)$.

As discussed in Section 2, the restrictions with respect to the degree of field non-uniformity which can be tolerated to ensure valid data are more severe for the $\bar{\alpha}(E, p)$ determination than for the Paschen curve measurement. However, from (11) and (12) it is possible to re-define $M$ in terms of the linear part of the Paschen curve as

$$
M=\frac{U_{0}}{\left(\frac{E}{p}\right)_{\lim }}
$$

i.e. $M$ can be determined from a single experiment.

As the value of $U_{0}$ is obtained by extrapolating from the $p d$ interval where the Paschen curve is linear, a small uncertainty in the slope of that section, which is itself $(E / p)_{\text {lim, }}$ can result in a large uncertainty in the evaluation of $U_{0}$, and might even lead erroneously to negative values for $U_{0}$. Consequently, to fully exploit the inherent advantages of this method, considerable experimental precision is essential. This aspect has been discussed previously in Section 2 .

$M$ values have been determined for many strongly electronegative gases and gas mixtures. However these data are as yet still dispersed among various conference publications, see e.g. [15-21]. However a preliminary assessment shows $\mathrm{SF}_{6}$ as exhibiting close to optimum insulation features.

With the introduction of $M$, it is possible to generalize the requirement for valid Paschen curve measurements, see Section 2.2.2, as [67]

$$
\left(\frac{p d}{M}\right)_{\max }=\frac{1}{\eta_{S}\left(\frac{E_{\max }}{E_{\text {lim }}}\right)-1}
$$

where $\eta_{S}$ is the field utilization factor, see (18). The left-hand side of (34) represents the maximum value of $p d / M$ for which the condition $z_{0}=d$ is upheld. For greater values of $p d / M, z_{0}<d$, resulting in invalid Paschen data. As the right-hand side of (34) contains only field parameters, this relation has been employed to determine the $(p d / M)_{\max }$ for different test gap geometries and assess the relevance of these [67].

Although the concept of $M$ was originally introduced in relation to electrode surface roughness, $M$ has a wider significance in that a general expression for discharge onset in a strongly electronegative gas/ gas mixture can be developed in terms of $M$ [12].

The electric field $E(z)$ along the field line associated with the maximum field strength $E_{\max }$ can be expressed as

$$
E(z)=E_{\max } f(z)
$$

in which $z$ is the distance from the electrode surface and the form of $f(z)$ is dependent upon the actual electrode geometry. By applying the streamer criterion, assuming a linear $\bar{\alpha} / p$ variation and utilizing (32), we can derive the following general expression for $E_{0} / p$ for any non-uniform field

$$
\frac{E_{0}}{p}=\frac{\frac{M}{p}+z_{0}}{\int_{0}^{z_{0}} f(z) d z}\left(\frac{E}{p}\right)_{\lim }
$$

As previously, $E_{0}$ is the value of $E_{\max }$ which satisfies the streamer criterion, and $z_{0}$ can be found from

$$
f\left(z_{0}\right)=\frac{E_{\mathrm{lim}}}{E_{\mathrm{o}}}
$$

Consequently if $f(z)$ is known, then it becomes possible to evaluate the discharge onset level of strongly electronegative gases solely from a knowledge of $(E / p)_{\lim }$ and the associated $M$. The application of this approach with respect to $\mathrm{SF}_{6} / \mathrm{N}_{2}$ mixtures is described in [12].

In general, however, a knowledge of $f(z)$ for a practical electrode with a rough surface will never be available, and thus a direct application of (36) to a practical system is not possible. Nevertheless, by 
making a comparative evaluation, this limitation can essentially be circumvented. Such an evaluation implies that a reference discharge characteristic, i.e. the $\left(U_{0}, p\right)$ characteristic, is available for the system in question. If in the same electrode system, the discharge onset levels $U_{\mathrm{oA}}$ and $U_{\mathrm{oB}}$ of two gases $\mathrm{A}$ and $\mathrm{B}$ are uniquely connected to electron avalanche growth along a particular line of force, then in such a situation $U_{\mathrm{oB}}$ is related uniquely to $U_{\mathrm{oA}} ;$ viz.

with

$$
U_{\mathrm{oB}}\left(p_{\mathrm{B}}\right)=\frac{M_{\mathrm{B}}}{M_{\mathrm{A}}} \frac{(E / p)_{\mathrm{B}_{\text {lim }}}}{(E / p)_{\mathrm{A}_{\mathrm{lim}}}} U_{\mathrm{oA}}\left(p_{\mathrm{A}}\right)
$$

$$
p_{B}=\frac{M_{\mathrm{B}}}{M_{\mathrm{A}}} p_{\mathrm{A}}
$$

Consequently, from a knowledge for gas $A$ of the functional dependence of $U_{\mathrm{oA}}$ with $p_{\mathrm{A}}$, the construction of a discharge onset characteristic for gas $B$ in the same practical system becomes a relatively simple matter, provided the relevant $M$ and $(E / p)_{l i m}$ data are available.

Full details of the derivation of (38) and (39) are given in [12], while experimental support for the comparative approach may be found in $[68,69]$.

\subsection{DETERMINATION OF THE FIELD ENHANCEMENT FACTOR $m$}

In the late 1980s, following a revival of interest in surface roughness effects through an EPRI project, it was realized that the original protrusion studies suggested an indirect method of determining the field enhancement factor for the surface of a practical conductor [70]. The essence of the method can be appreciated from Figure 10, which indicates that as $\left(p / H_{\mathrm{i}}\right) \rightarrow \infty, \xi$ tends asymptotically to some limiting value, $\xi_{\text {limn }}$. In [70], it is shown theoretically that in fact

$$
\xi_{\lim }=\frac{1}{m}
$$

Confirmation of this fact can be obtained from (29), which can be inverted to yield an explicit expression for $\xi$, viz.

$$
\xi=\frac{2}{3}\left(1-\sin \left(\frac{\theta}{3}\right)\right)
$$

with

$$
\theta=\arcsin \left[2\left(1-\frac{H_{\mathrm{j}} M}{p}\right)^{3}-1\right]
$$

where $M$ has replaced $K / \bar{\kappa}$. From (42), it is evident that, as $p / H_{\mathrm{i}} \rightarrow$ $\infty, \theta \rightarrow \pi / 2$ and thus $\xi \rightarrow \frac{1}{3}$, in agreement with the $m$ value for a hemispherical protrusion, see also Figure 10.

The method is based upon the measurement of the discharge onset voltage $U_{0}$ associated with the surface in question for a range of gas pressures. The $U_{0}$ data subsequently are analyzed to provide $\xi$ data. To obtain an asymptotic limit for $\xi$, it is desirable to undertake $U_{0}$ measurements over an extensive pressure range. In reality however, this is not possible. In practice, $U_{0}$ measurements will be confined to the pressure range, $0.1<p / \mathrm{MPa}<1$. Such a limited pressure range implies that a regression analysis of the $\xi$ data is necessary. Full details of the procedure adopted can be found in [70]. To date the method has been applied to both machined [70] and sand-blasted electrodes [71].

\subsection{DISCUSSION}

From a study of the discharge onset conditions of roughness models, it was possible to gain an appreciation of the significance of the field perturbation resulting from surface roughness. By considering many protrusion models, it became evident that the reduction in insulation strength is controlled by both the field enhancement and the associated field distribution. Because the models are very primitive representations of a practical rough surface, these models cannot be used to predict either the breakdown voltage in a practical systern, or, conversely, the actual dimensions of the actual surface protrusion:s.

Nevertheless, the modeling of surface roughness led directly to the concept of a figure of merit $M$ for a strongly electronegative gas/gas mixture. This parameter was subsequently shown to have direct applicability with respect to the discharge onset conditions for such gases. This enables a rapid assessment of the insulation capabilities of replacement gases to be made, prior to undertaking additional laboratory tests.

Finally, surface roughness modeling provided the impetus for the development of an experimental method to determine the field enhancement factor $m$ of a practical rough surface.

\section{5 .INDUCED CHARGE, $\lambda$ AND $\phi$ FUNCTIONS}

\subsection{INTRODUCTION}

In insulation studies measurements of transient currents or charge are undertaken at a detecting electrode. Such measurements are in effect measurements of the charge induced on that electrode by the charge/discharge event of interest. Although the phenomenon of 'electricification' by electrostatic induction has been know since the work of Faraday in the 1830s, it is only with the introduction of the $\lambda$ and $\phi$ functions that it has become possible to evaluate induced charge quantitatively in general situations with polarizable media [72-74]. Initial studies were restricted to systems in which polarizable media were absent, see reference to those in $[14,75-77]$.

These two functions were introduced in the second half of the $1980 \mathrm{~s}$ as a means of quantifying the charge induced on an electrode by a source of charge. Subsequently, a full mathematical derivation of the functions was provided [76]. To-date the functions have been employed principally in studies of electrostatic probe measurements and partial discharge transients. These topics are discussed in Sections 6 and 7 below, and only the theoretical basis of the functions will be discussed in this Section.

\subsection{INDUCED CHARGE}

Usually, the concept of induced charge is discussed in qualitative terms. However prior to discussing its quantitative evaluation it is necessary to comment on some aspects of the phenomenon. This can be readily done with reference to a standard problem of electrostatics.

Consider an isolated electrode which is initially in a field-free environment: i.e. the electrode is at zero potential and carries zero net charge. The surface charge density $\sigma$ also is zero. A charge source is then located at a known distance from this electrode. This situation is illustrated in Figure 13a for a spherical conductor and a point charge. 
For this situation, the field solution indicates that now $\sigma \neq 0$. However, on evaluating the charge $q$ induced by the source charge $Q$, we find that

$$
q=\int \sigma d A=0
$$

Nevertheless there has been a change in electrode potential. This change $\Delta U$ is given by

$$
\Delta U=\frac{Q}{4 \pi \varepsilon b}
$$

which may also be expressed as

$$
\Delta U=\frac{\frac{R}{b} Q}{C}
$$

where $C$ is the capacitance of the isolated sphere: i.e. the change in sphere potential is associated with a charge $(R / b) Q$. This charge has been named the Laplacian induced charge qu [77], i.e.

$$
\Delta U=\frac{q \mathrm{~L}}{C}
$$

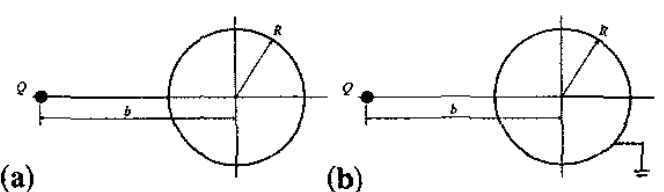

Figure 13. Characteristics of induced charge.

The sphere is now grounded, see Figure $13 \mathrm{~b}$, i.e. $\Delta U=0$. On solving for the new field condition, we still find that $\sigma \neq 0$, but in contrast to the previous situation for which $q=0$, we now have

$$
q=\int \sigma d A=-\frac{R Q}{b}
$$

This finite value of $q$ has been called the Poissonian induced charge $q_{\mathrm{P}}$ [77]. The situation implies that the grounding of the electrode results in the transfer of charge $\Delta Q$ to the electrode: i.e. the Laplacian induced charge can also be associated with a charge transfer, viz.

$$
q_{\mathrm{L}}+\Delta Q=0
$$

The initial values of the Poissonian induced charge and the Laplacian induced charge are numerically equal, but of opposite polarity. The fact that the latter component of the induced charge can be associated with both a change in potential and a transfer of charge is the basis of induced charge measurements.

The above concepts have been generalized by introducing the fol lowing definitions [77]. The Poissonian induced charge is that component of the induced charge related to the Poissonian field established by the space charge. The Laplacian induced charge is that component of the induced charge related to the Laplace field associated with the change in conductor potential.

\subsection{DEFINITION OF THE $\lambda$-FUNCTION}

A point charge $d Q$ located in a heterogeneous dielectric system in the presence of electrodes will electrostatically induce charge on all electrodes. From basic field theory, it is clear that the charge induced on an electrode by $d Q$ depends in a unique way upon the location and magnitude of this charge. With reference to the $i$ th electrode in an $N$ electrode system, the Poissonian induced charge $d q_{\mathrm{P} i}$ related to $d Q$ may be expressed as

$$
d q_{\mathrm{Pi}}=-\lambda_{i} d Q
$$

$d q_{\mathrm{Pi}}$ also includes the contribution due to changes in the dielectric polarization related to the field of $d Q$. The parameter $\lambda_{i}$ is simply a dimensionless factor of proportionality. In general for a system with $N$ electrodes, we need $N$ different $\lambda$ functions, one for each electrode. This is necessary when the charge induced by the same source differs from electrode to electrode.

The total Poissonian induced charge on the $i$ th electrode associated with a distribution of both volume space charges and charges on dielectric interfaces is thus the sum of the contributions arising from all such space elements $d \Omega$ and surface elements $d S$ of charge, i.e.

$$
q_{\mathrm{P} i}=-\iiint_{\Omega} \lambda_{i} \rho d \Omega-\iint_{S} \lambda_{i} \sigma d S
$$

The volume charge density $\rho$ and the surface charge density $\sigma$ are functions of location and these may also vary with time, but $\lambda_{i}$ is independent of time. The volume integral is extended to all space between the electrodes, and the surface integral to all interfaces $S$ between dielectrics.

The $\lambda_{i}$ function has the value unity at the surface of the $i$ th electrode and its value tends to zero as the spatial location recedes from this electrode, and also as it approaches all other electrodes. At the surface of these electrodes its value is zero. Equation (50) is the defining equation for $\lambda_{i}$ and as such is employed to determine the properties of the function.

Using (50), Maxwell's equations and vector analysis, it has been shown in [76] that the $\lambda_{i}$ function is a solution of the general Laplace equation for the complete system geometry, viz.

$$
\vec{\nabla} \cdot\left(\varepsilon \vec{\nabla} \lambda_{i}\right)=0
$$

The boundary conditions are $\lambda_{i}=1$ at the $i$ th electrode and $\lambda_{i}=$ 0 at all other electrodes. In addition, at dielectric interfaces between two media, the normal derivatives of $\lambda_{i}$ must obey the condition

$$
\varepsilon_{+}\left(\frac{\partial \lambda_{i}}{\partial n}\right)_{+}=\varepsilon_{-}\left(\frac{\partial \lambda_{i}}{\partial n}\right)_{-}
$$

where the + and - signs refer to the opposite sides of the interface.

As (51) is Laplace's equation, any standard method of solving can be employed to evaluate the variation of $\lambda_{i}$. However, although the $\lambda_{i}$ function is a solution of Laplace's equation, this fact does not imply that $\lambda_{i}$ is a potential or a voltage.

\subsection{DEFINITION OF THE $\phi$-FUNCTION}

The influence of the dielectric polarization $\vec{P}$ upon the magnitude of the induced charge remains concealed in the $\lambda$ function. However, introducing $\vec{P}$ explicitly into the analysis allows its influence to be taken into account. In such an approach the entire interelectrode volume is considered to be vacuum, in which a distribution of dipoles with a dipole moment density $\vec{P}$ represents the actual dielectric. Thus $\vec{P}$ may be related to a point dipole of moment $d \vec{p}$, where 


$$
d \vec{p}=\vec{P} d \Omega
$$

and where $d \Omega$ represents a volume element.

The Poissonian induced charge on an electrode is now considered to consist of two distinct components, viz. one linked specifically with the space charge distribution, and a second component directly linked to the dipoles representing the dielectric. The effective polarization $\vec{P}_{\mathrm{e}}$ of a solid dielectric will depend on the applied voltage, and on the field perturbation caused by the space charge distribution, i.e.

$$
\vec{P}=\vec{P}+\delta \vec{P}
$$

where $\vec{P}$ is linked exclusively with the applied voltage, and $\delta \vec{P}$ is associated with the presence of the space charge distribution.

As above, consider a point charge $d Q$ placed within the interelectrode volume. If $d q_{i Q}$ represents the induced charge on the $i$ th electrode resulting from $d Q$, then we have

$$
d q_{i Q}=-\phi_{i} d Q
$$

where $\phi_{i}$ denotes the proportionality factor between $d q_{i Q}$ and $d Q$. In addition the induced charge $d q_{i p o l}$ on the $i$ th electrode due to a dipole moment $d \vec{p}$ is given by $[72,75,77]$

$$
d q_{\text {ipol }}=-d \vec{p} \cdot \vec{\nabla} \phi_{i}
$$

Individually, the expressions given in (55) and (56) represent the two components of the induced charge discussed above. Hence, the resulting Poissonian induced charge on the $i$ th electrode can, with the dipole representation, be expressed as

$$
\begin{gathered}
q_{\mathrm{P} i}=-\iiint_{\Omega}\left(\phi_{i} \rho+\delta \vec{P} \cdot \vec{\nabla} \phi_{i}\right) d \Omega-\iint_{S} \phi_{i} \sigma d S \\
\begin{array}{c}
\text { field analysis } \\
\text { of } \\
\text { dielectric system }
\end{array}
\end{gathered}
$$

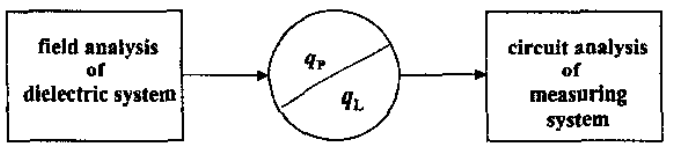

Figure 14. Interface between field analysis and circuit analysis. Here $q_{\mathrm{P}}$ is the Poissonian induced charge, $q_{\mathrm{L}}$ the Laplacian induced charge, and $q_{\mathrm{P}}=-q_{\mathrm{L}}$.

As previously, the volume integral is extended over the entire volume external to the $N$ electrodes and the surface integral to all dielectric interfaces $S$. The integral expressions for $q_{\mathrm{P} i},(50)$ and (57), are equivalent.

Similar to the $\lambda_{i}$ function, $\phi_{i}$ takes the value unity at the surface of the $i$ th electrode, while its value is zero at all other electrodes. Moreover (57) represents the defining equation for $\phi_{i}$.

With (57) it is possible to establish the functional basis for $\phi_{i}$. In [76] the $\phi_{i}$ function is shown to be a solution of the reduced Laplace equation, viz.

$$
\nabla^{2} \phi_{i}=0
$$

The boundary conditions are $\phi_{i}=1$ at the surface of the $i$ th electrode and $\phi_{i}=0$ at all other electrodes.

\subsection{DISCUSSION}

The existence of a charge in/on a dielectric medium leads to an induced charge on a detecting electrode through the phenomenon of electricification by electrostatic induction. The induced charge can be mathematically resolved into two components. The major benefit of this procedure is that it provides an interface between the field and circuit analyzes of the problem under study, see Figure 14.

The Poissonian induced charge is infrangibly linked with the source charge, and remains so long as the source charge exists. This induced charge component can be quantified relative to the source charge through either the $\lambda$ function or the $\phi$ function. The Laplacian induced charge is the primary source for the measuring system, ard hence it is this component which we can manipulate through the impedance of the external circuit: i.e. we measure either the potential which the isolated detecting electrode attains, or the current flow (charge transfer) through the electrode ground lead.

The $\lambda$ function is related to the $\vec{D}$ field and thus is linked directly to the space charge: i.e. this function takes account of cielectric polarization implicitly. In contrast the $\phi$ function is related to the $\vec{P}$ field, i.e. the function deals with dielectric polarization explicitly. Hence it becomes possible to distinguish between the influence of the space charge itself and that of the dielectric polarization upon the induced charge. By this means the significance of changes in polarization $(\delta \vec{P})$ in contributing to the induced charge can be deduced.

In practice the direct application of the $\phi$ function is limited, because a knowledge of $\delta \vec{P}$ requires a prior knowledge of the field perturbation produced by the space charge. However, as this source term itself is an unknown quantity, the associated field perturbation cannot be determined. Nevertheless, the $\vec{P}$ field approach has great relevance for analytical studies in which the source term is pre-defined. In contrast, the $\lambda$ function can be applied readily to practical systems.

\section{ELECTROSTATIC PROBES 6.1 INTRODUCTION}

Since the late 1960 s, electrostatic probe measurements traditionally have been interpreted with reference to a capacitive equivalent circuit, e.g. see [78-82], in which a capacitance is considered to exist between the sensor plate and the solid dielectric surface, see Figure 15. This is a straightforward violation of the definition of capacitance. Hence such a capacitance does not exist. Unfortunately, the misuse of the concept of capacitance is a common occurrence in insulation/discharge studies, e.g. we have void capacitance, corona capacitance, leader capacitance, all of which are misconceptions.

An approximate evaluation of probe measurements may of course be undertaken by employing a physically valid assumption, viz. that the field associated with the practical measurement is uniform. However, the limits of the validity of such an assumption have yet to be quantitatively established.

We will now demonstrate how the $\lambda$ function can be employed to evaluate probe measurements correctly, without violating basic definitions, and also to deduce the response characteristics of probes.

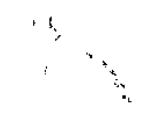




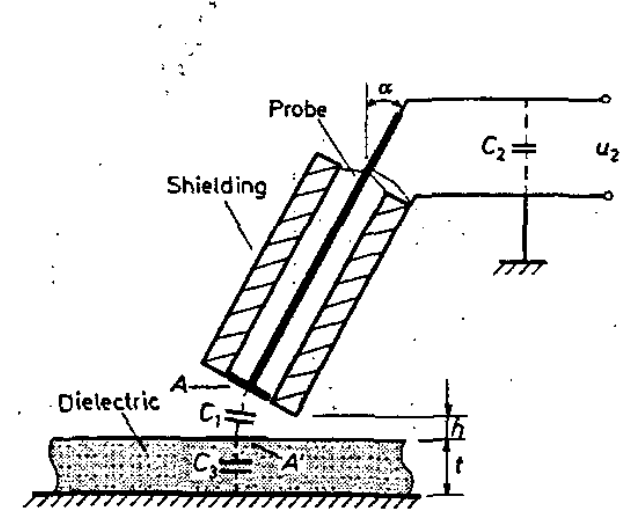

Figure 15. Probe capacitance [82].

\subsection{EVALUATION OF PROBE MEASUREMENTS}

In the mid-1980s, with the development of HVDC-GIS, the limitations of an evaluation based upon the traditional capacitance approach were becoming all too obvious. It was realized that a correct quantitative interpretation of probe measurements required a proper field solution which considered the entire dielectric surface and system boundaries $[73,83-85]$. The basis for a field theoretical approach had however been published in the mid-1970s [86], but unfortunately that study was overlooked.

On the theory of probe measurements, only two of the published proposals have been developed fully $[87,88]$, and thus discussion will be restricted to these. Furthermore, because the two approaches are effectively equivalent, it is only necessary to discuss one in any detail, and to illustrate the procedure involved, before referring to the differences that distinguish the two approaches.

The existence of a charge at a dielectric surface gives rise to electricification by electrostatic induction of the probe. If the volume charge density within the solid dielectric is assumed to be zero, then with respect to the contributions from all surface elements of charge we have

$$
q=-\iint_{A_{0}} \lambda \sigma d A
$$

in which $q$ is the total Poissonian induced charge on the sensor plate, and $\sigma$ is the surface charge density on the surface element $d A$ of $A_{0}$, the surface of the solid dielectric. It should be noted that only the Poissonian component of the induced charge will be discussed in Section 6 . Thus it is convenient to omit the identifying subscript $P$ in this Section. As described in Section 5, if charge existed within the bulk of the solid dielectric, (59) would contain an additional term.

To evaluate the unknown charge distribution requires the solution of the basic integral (59). To do so it is necessary to discretize both the charged surface area and the probe scanning procedure. Thus the basic measuring technique requires a multipoint measurement to be performed with the probe. This scanning procedure generates a series of probe responses $p_{i}$, each of which is related to the specific position $i$ of the probe relative to the selected origin on the dielectric surface.

\subsection{1 $\Lambda$ MATRIX AND EVALUATION PROCEDURE}

With a sufficient degree of discretization of the surface area under study, each of the sub-areas $A_{j}$, with $j=1 \ldots n$, may be considered as uniformly charged with a surface density $\sigma_{j}$ : i.e. in effect $\sigma_{j}$ represents the average charge density of $A_{j}$. Hence the charge induced on the probe by a single sub-area of charge can be expressed as

$$
q=-\sigma_{j} \iint_{A_{j}} \lambda d A
$$

As the integral in (60) contains only $\lambda$ and surface area, we are able to take into account simultaneously both the geometrical scanning procedure and the relevant $\lambda$ function by introducing a $\Lambda$ matrix. The formation of this matrix must correspond with the number of sub-areas $n$. For each probe position, a sub-matrix $\Lambda_{i}$ is derived with $1 \times n$ elements, i.e. a row matrix. Each element is given by

$$
\Lambda_{i j}=\iint_{A_{j}} \lambda_{i} d A
$$

where $d A$ is a surface element of $A_{j}$. The index $i$ refers to the position of the probe, while $j$ relates to the surface area in question. The $i$ in $\lambda_{i}$ indicates that the values to be associated with a specific sub-area are connected with the discretization of the charged surface area, and because the $\lambda$ function itself is dimensionless, the dimension of the $\Lambda$ matrix is that of area.

With the probe-fixed in a position directly above sub-area $A_{i}$, the contribution to the probe response (induced charge) from surface charge element $\sigma_{j}$ of sub-area $A_{j}$ is given by $\Lambda_{i j} \sigma_{j}$. By summation of all contributions for $j=1 \ldots n$, the total probe response $p_{i}$ is obtained for the probe positioned above $A_{i}$, i.e.

$$
p_{i}=\sum_{j=1}^{n} p_{i j}=\sum_{j=1}^{n} \Lambda_{i j} \sigma_{j}
$$

Because the $\sigma_{j}$ can be expressed as an $n \times 1$ column matrix, this implies that (62) also can be expressed in matrix form, viz.

$$
\mathbf{p}_{i}=\boldsymbol{\Lambda}_{i} \boldsymbol{\sigma}_{j}
$$

where $\boldsymbol{\Lambda}_{i}$ represents a $1 \times n$ row matrix.

The global probe response is developed by positioning the probe above each sub-area in turn, i.e. for $i=1 \ldots n$. To solve for all $\sigma_{j}$ utilizing the series of probe responses $p_{i}$, the $n \Lambda_{i}$ sub-matrices are combined into a $n \times n$ matrix. Thus on the basis of (63), the global response is given by

$$
\mathbf{p}=\Lambda \boldsymbol{\sigma}
$$

where $\mathbf{p}$ is an $n \times 1$ column matrix, in which each element denotes the measured total induced charge on the probe sensor plate for each position of the probe. $\sigma$ is also a $n \times 1$ column matrix with each element representing the surface charge density on a sub-area. For a given $\Lambda$ matrix and a measured set of probe responses, (64) can be solved for the unknown $\sigma_{j}$ by inverting the matrix, i.e. (64) may be re-written as

$$
\boldsymbol{\sigma}=\boldsymbol{\Lambda}^{-1} \mathbf{p}
$$

The number of surface elements and the number of probe measurements must agree. The reason for this rigidity is that, for a given set of probe responses, (65) has one and only one solution. 
A full description of the application of this method of analysis to an experimental situation is given in [87].

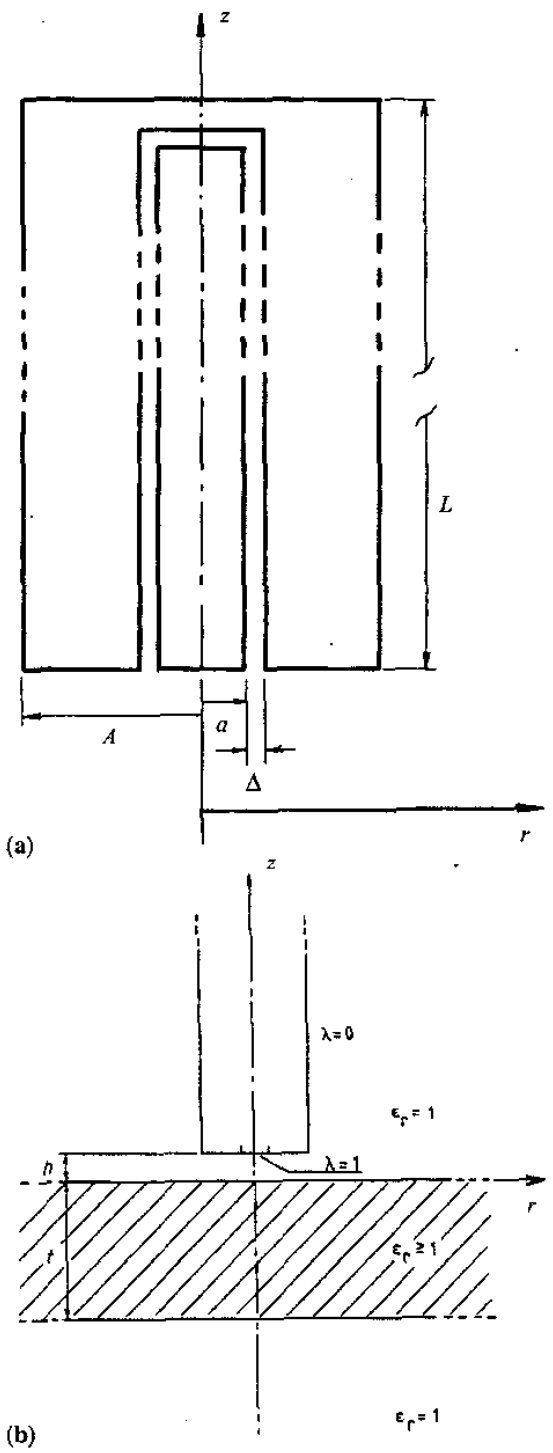

Figure 16. Free probe geometry.

\subsubsection{PROBE RESPONSE}

Following the global evaluation of $\sigma_{j}$ from (65), it is possible to obtain a deeper insight into the total probe response $p_{i}$ by inserting the derived values of $\sigma_{j}$ into (62). In this way, the individual induced charge contributions $\Lambda_{i j} \sigma_{j}$ can be identified. With this procedure, it may be demonstrated that areas of charge not directly beneath the - probe can contribute a significant percentage of the probe signal, see [87]. Previously, such a feature had been demonstrated experimentally by Yashima et al. [84].

\subsubsection{ALTERNATIVE PROCEDURE}

A similar field approach has been developed by Takuma and colleagues $[84,88]$. This also requires the solution of a matrix equation, the terms of which are expressed as [88]

$$
W_{p}(I)=\sum_{J=1}^{N} A(I, J) \sigma(J)
$$

where $W_{p}(I)$ is the probe response at a measuring position $I, \sigma(J)$ the charge density at a subarea $J$, and $N$ is the total number of subareas, i.e. (66) is equivalent to (62). The coefficient $A(I, J)$ is the response at position $I$ due to a unit charge density at subarea $J$. Each coefficient $A(I, J)$ is then the ratio of the induced charge to the source charge. This coefficient is dependent on the complete systern geometry and is determined by numerical field calculation.

As the dielectric surface is discretized into $N$ subareas, $A(I, J)$ is evaluated $N$ times for each of the $N$ positions of the probe. In [88] however, the authors indicate how to reduce the number of calculations from $N^{2}$ to $N$. With the $\lambda$ function approach, $\lambda_{i}$ would be evaluated once for each of the $n$ probe positions and thereafter $\Lambda_{i j}$ determined for each of the $n$ subareas. Hence, although the procedures for determining the $A$ and $\Lambda$ matrices differ, the two matrices are identical and thus the two approaches are equivalent.

In a subsequent study, Takuma and Hamada have numerically compared the two main methods of evaluating surface charge density [89]. This study indicates that considerable care is required in implementing the numerical procedures, particularly with respect to geometric discontinuities.

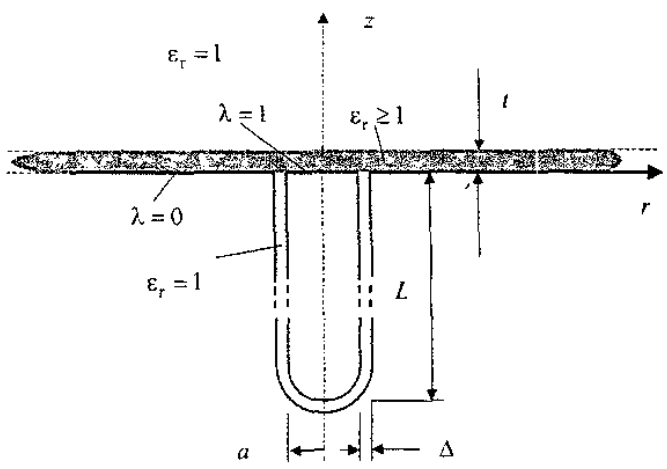

Figure 17. Bound probe geometry.

\subsection{PROBE CHARACTERISTICS}

On the basis of (59), it is possible to examine the response of a probe to a known surface charge distribution. By this means the characteristics of probe designs can be readily evaluated and compared. Such studies have been undertaken for both free [90-93] and bound [94-96] probes.

A free probe is one which has freedom of movement with respect to the charged surface. In general such probes consist of a long cylindrical shaft at ground potential, with a coaxial circular sensor disc mounted at one end, see Figure 16. This disc is insulated from the shaft, which acts 

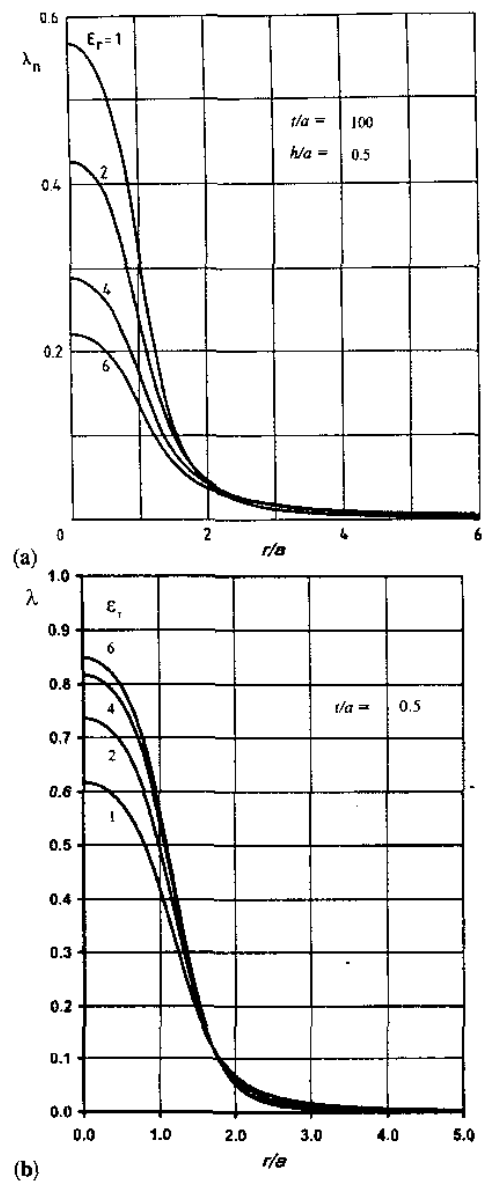

Figure 18. Variation of $\lambda$ along a dielectric interface: (a) free probe (near interface); (b) bound probe.

as a guard ring. With free probes it is possible to implement a scanning procedure whereby the probe is moved parallel to the charged surface. For the situation shown in Figure 16, there are two gas/solid dielectric interfaces. To distinguish between these, we will refer to the 'near' interface and the 'far' interface. There is a $\lambda$ distribution associated with each.

A bound probe is an integral part of an electrode and thus such probes lack freedom of movement with respect to the charged surface: For planar dielectric slabs or sheets in contact with a planar electrode, the probe sensor plate is mounted flush in this electrode, see Figure 17, but is insulated from the planar electrode. Bound probes have been used to investigate the conditions associated with discharge development along the surface of a charged gaseous/solid-dielectric interface $[97,98]$, and also the charge which has accumulated on the side of a right cylindrical spacer in vacuum $[99,100]$. Since the mid-1960s, bound probes have been employed widely in gas discharge studies to record the temporal variation of the field during discharge growth, see Meek and Craggs [101].

Typical variations of the $\lambda$ functions along the dielectric interface for free and bound probes are shown in Figure 18. The $\lambda$ function for the free probe relates to the near interface. Each probe exhibits a bell shaped distribution, with the maximum value occurring on the axis $(r=-0)$. However for increasing values of the solid dielectric permittivity, the probes display contrasting behavior in that, for the free probe, a marked reduction in $\lambda(0)$ is observed, while for the bound probe $\lambda(0)$ increases. With respect to the planar dielectric of Figure 16, the far interface $\lambda$ function is strongly influenced by the thickness of the dielectric $[90,91]$.

The effect of the probe geometry and system geometry upon $\lambda$ have been discussed fully [90-94]. However, to assess the significance of changes in $\lambda$ quantitatively, it is necessary to introduce other parameters which describe the detection sensitivity and the spatial resolution.

If $r, z$ represent cylindrical coordinates, see Figures 16 and 17, then for a circular disc of constant surface density $\sigma_{0}$ located at a dielectric interface and coaxial with the probe axis, the induced charge $q$ on the sensor plate is given by

$$
q(r)=-2 \pi \sigma_{0} \int_{0}^{r} \lambda\left(r^{\prime}, z\right) r^{\prime} d r^{\prime}
$$

where $r^{\prime}$ is a dummy variable, and a constant $z$ represents a planar interface. If $Q$ represents the actual charge on the dielectric interface directly beneath/above the probe sensor plate, i.e.

$$
Q=\pi a^{2} \sigma_{0}
$$

where $a$ denotes the radius of the probe sensor, see Figures 16 and 17 , then a detection sensitivity $S_{\mathrm{e}}(r)$ may be defined as

$$
S_{\mathrm{e}}(r) \equiv\left|\frac{q(r)}{Q}\right|
$$

Hence by utilizing (67) and (68) we obtain

$$
S_{\mathrm{e}}(r)=\frac{2}{a^{2}} \int \lambda\left(r^{\prime}, z\right) r^{\prime} d r^{\prime}
$$

From a study of $S_{\mathrm{e}}$, see [90-94], it is demonstrated that areas of charge outside that immediately subtended by the probe can account for a major portion of the induced charge signal.

With respect to $q$, it is also possible to define a spatial selectivity factor $S_{\mathrm{p}}(r)$ as follows

$$
S_{\mathrm{p}}(r)=\frac{\int_{0}^{r} \lambda\left(r^{\prime}, z\right) r^{\prime} d r^{\prime}}{\int_{0}^{r_{0}} \lambda(r, z) r d r}
$$

or

$$
S_{\mathrm{p}}(r)=\frac{S_{\mathrm{e}}(r)}{S_{\mathrm{e}}\left(r_{\mathrm{o}}\right)}
$$

where $r_{0}$ is the maximum radial extent of the charged area. Again a constant charge density $\sigma_{0}$ is assumed to exist at the interface. The parameter $r_{0}$ allows the spatial selectivity of the probe to be quantified. With this parameter it has been possible to demonstrate the importance of a local $(\lambda=0)$ electrode boundary $[90,91]$. The effect of this boundary is to suppress the induced charge contributions from non-local areas of charge and thus the introduction of such boundaries can be used to optimize the spatial selectivity of the probe.

The probe response with respect to both non-uniform charge distributions and eccentric charge distributions is discussed in $[95,96]$, respectively. 


\subsection{DISCUSSION}

Through the use of the $\lambda$ function, it has been possible to develop a method to evaluate electrostatic probe measurements. Although dependent on the position of the probe in the overall geometry, $\lambda$ however is independent of the discretization of the charged surface area. This feature simplifies the evaluation of the $\Lambda$ matrix, the geometric matrix, associated with the subareas. This simplification is advantageous as it is through this matrix, together with that of the measured probe responses, that the unknown surface charge density is determined.

The introduction of the $\Lambda$ matrix also enables the details of the probe response to each surrounding distribution of charge to be subsequently examined.

The $\lambda$ function also allows the probe response for known surface charge distributions to be examined with reference to probe geometry and system geometry. By this means an appreciation of the characteristics of electrostatic probes can be gained.

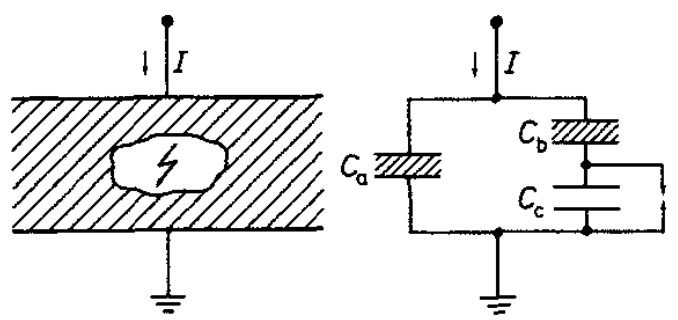

Figure 19. The equivalent circuit approach.

\section{PARTIAL DISCHARGE TRANSIENTS}

\subsection{INTRODUCTION}

In contrast to partial discharge measurements, the subject of partial discharge transients had, up until the mid-1980s, essentially stagnated in a simplistic circuit theory approach: a valid physical theory linking the transient signal at the system terminals with the partial discharge event did not exist. This static situation occurred because of the fixation with the concept of void capacitance, and consequently the use of circuit theory to address what is in essence a field problem. This concept is still so ingrained that, ten years after the fallacy behind this concept had been highlighted [102], it continues to be included in the second edition of Kuffel and Zaengl [8]. In an attempt to justify the use of capacitance, this new edition contains some irrelevant comments about field lines. These comments serve no purpose other than to confuse further the whole issue of what is and what is not a capacitance.

\subsubsection{THE STATUS QUO}

For very many years, an equivalent circuit approach was used when discussing partial discharges, see Figure 19. With respect to this circuit, $C_{\mathrm{a}}$ denotes the capacitance of the system; $C_{\mathrm{b}}$ is considered to be the capacitance of the solid dielectric in series with the void, while $C_{\mathrm{c}}$ is considered to be the capacitance of the void. The gap across $C_{\mathrm{c}}$ indicates that the gas-filled void breaks down when there is sufficiently high field strength in the void.

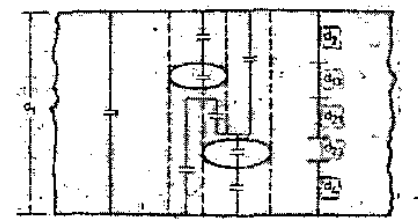

(a)

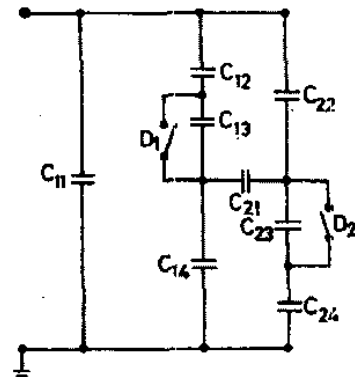

(b)

Figure 20. Lumped capacitance model of a dielectric with two voids: adapted from $[105,106]$. (a) Solid dielectric with two voids, (b) Equivalent circuit.

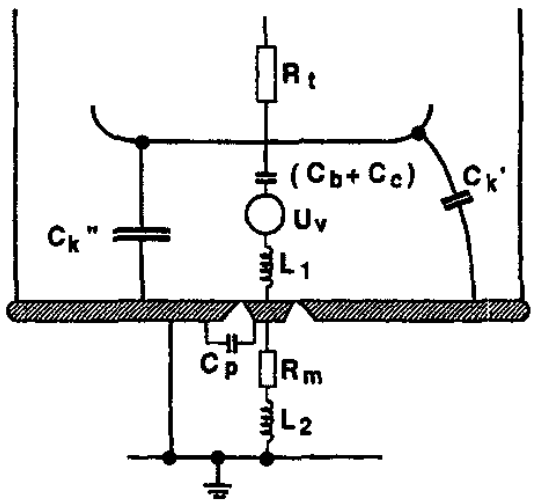

Figure 21. Equivalent circuit incorporating void inductance [107$109]$.

An equivalent circuit is any circuit which can generate, as faithfully as possible, the signals which are manifest at the terminals of the actual system. In this context, the system is the proverbial black-box. With respect to Figure 19, this simple equivalent circuit has been very successful in promoting the development of methods for partial discharge detection, see $[103,104]$. Unfortunately, this success has lead to the identification of the capacitive circuit with the actual dielectric system. It has however been forgotten that the operation of an equivalent circuit need in no way correspond to the physical processes which occur in the actual system. With respect to the partial discharge phenomenon, this limitation of equivalent circuits has apparently been overlooked during the past 30 years.

The propensity to treat insulation problems in terms of circuit theory can lead to absurd representations; viz. the multi-capacitance model of Agoris and Hatziargyriou [105,106], see Figure 20, while Danikas introduced a void inductance [107-109], see Figure 21. These approaches certainly enhance the black-box concept.

Although the dielectric system as a whole has a capacitance, the basic fact is that $C_{\mathrm{b}}$ and $C_{\mathrm{c}}$ do not exist, even in a mathematical sense for a Laplace field, i.e. with no space charge present. Such a situation lead Pedersen to consider a field approach based on the concept of induced charge [72,74]. 


\subsection{PARTIAL DISCHARGES AND INDUCED CHARGE}

The occurrence of a partial discharge in a gaseous void, in a bulk dielectric, creates charge in the form of electrons, positive ions and possibly also negative ions. Such charge automatically will induce charge on all electrodes. For those electrodes not at ground potential, the induced charge will bring about a change in potential of these electrodes. The consequences of this situation are discussed in Section 7.3.3 with respect to partial discharge measurements.

With reference to the measuring electrode, it is evident from Section 5 that the $\lambda$ function can be used to evaluate the Poissonian induced charge. The source charge is the partial discharge which has occurred in a closed volume, resulting in a deposition of charge on the void wall. Within this confined space, the net.charge produced by the discharge will be zero. Hence we can represent the charge distribution by a system of multipoles [75].

Moreover, if the dimensions of the void are such that $\vec{\nabla} \lambda$ may be assumed constant within the void, then it is only necessary to consider the dipole moment of the charge distribution within the void. This $\vec{\nabla} \lambda$ assumption implies that the second and higher order derivatives of $\lambda$ do not exist, and thus the second and higher order multipoles will not contribute to the induced charge.

If $\vec{\nabla} \lambda$ is not constant within the void, then it becomes necessary to consider if the induced charge contributions from these multipoles are significant in comparison to the dipole contribution. A non-uniform $\vec{\nabla} \lambda$ within the void will occur if the spatial non-uniformity of $\lambda$ external to the void is comparable with the dimensions of the void, or if the void is in the proximity of an interface between two media.

The dipole moment $\vec{\mu}$ of a distribution of charge is given by [110]

$$
\vec{\mu}=\int \vec{r} d Q
$$

where $\vec{r}$ is a radius vector which locates the position of the charge element $d Q$. As we are principally interested in the final value of the induced charge on the measuring electrode, the dipole integral reduces to one involving the surface charge density $\sigma$ at the void wall; i.e. we have

$$
d Q=\sigma d A
$$

where $d A$ represents a surface element.

From (73) and (74) it is evident that $\vec{\mu}$ is controlled by the spatial extent and distribution of $\sigma$, see also [111]. Hence, in analytical studies, the magnitude of $\vec{\mu}$ is dependent on the assumptions made with respect to the partial discharge development.

By considering the induced charge associated with two point charges of opposite polarity, separated by an infinitesimal distance, it can be readily shown that the Poissonian induced charge $q_{\mathrm{p}}$ associated with a dipole $\vec{\mu}$ is given by $[74,75]$

$$
q_{p}=-\vec{\mu} \cdot \vec{\nabla} \lambda
$$

For small voids, $\vec{\nabla} \lambda$ may be taken as constant. This situation allows the introduction of another function, $\lambda_{0}$, which represents the $\lambda$ function for the void-free system $[75,112]$. By mathematical analogy with electrostatic fields, the relation between $\lambda$ and $\lambda_{0}$ may be expressed as

$$
\vec{\nabla} \lambda=h \vec{\nabla} \lambda_{0}
$$

where for the void geometries under consideration, $h$ is a constant dependent on the void geometry and the relative permittivity $\varepsilon_{r}$ of the bulk dielectric, e.g. for a spherical void

$$
h=\frac{3 \varepsilon_{r}}{1+2 \varepsilon_{r}}
$$

With the introduction of $\lambda_{0}$, the Poissonian induced charge from a dipole is given by

$$
q_{\mathrm{P}}=-h \vec{\mu} \cdot \vec{\nabla} \lambda_{0}
$$

\subsection{FACTORS INFLUENCING INDUCED CHARGE}

\subsubsection{VOID PARAMETERS}

With the adoption of a dipole representation, it became possible to examine the influence of the void parameters upon the induced charge. However this required the evaluation of the source dipole moment. This moment is related to the void wall charge, which establishes a field within the void opposing the applied field. If the net field in the void is reduced sufficiently, all discharge development ceases and the dipole moment is constant in time. As an initial approach, it was assumed that the whole void participated in the discharge, and that, even after the discharge, the void field remained uniform. Even if these assumptions are not fulfilled in practice, the general conclusions which can be drawn from the analysis will remain valid.

From the assumptions, it was possible to derive an expression for the dipole moment of a partial discharge in an ellipsoidal void [112], viz.

$$
\vec{\mu}=\frac{K}{h} \varepsilon \Omega\left(\vec{E}_{\mathrm{i}}-\vec{E}_{l}\right)
$$

where $K$ is a dimensionless parameter which is dependent upon the geometry of the ellipsoid. $\Omega$ is the ellipsoid volume, while $\varepsilon$ is the permittivity of the bulk dielectric. The field strengths $E_{\mathrm{i}}$ and $E_{l}$ represent the inception field strength for discharge development, and the limiting field strength for ionization growth, respectively. That is, a partial discharge can develop when the void field attains a value of $E_{\mathrm{i}}$ and will be quenched when the field is reduced to $E_{l}$. Insertion of (79) in (78) enables the Poissonian induced charge to be expressed as

$$
q_{\mathrm{P}}=-K \varepsilon \Omega\left(\vec{E}_{\mathrm{i}}-\vec{E}_{l}\right) \cdot \vec{\nabla} \lambda_{0}
$$

The difference $\left(\vec{E}_{\mathrm{i}}-\vec{E}_{l}\right)$ was quantified by using the streamer criterion. In doing so, it is implicitly assumed that the void dimensions and the gas pressure are such that swarm conditions could be established. Such conditions are necessary to ensure the validity of the use of the macroscopic gaseous ionization coefficients.

Examination of (80) indicates that the Poissonian induced charge is dependent on void location, void geometry, void physical dimensions, void gas, void gas pressure, and the permittivity of the (homogeneous) bulk dielectric. Unfortunately, a knowledge of all of the above parameters can only be available for measurements made under controlled laboratory conditions. Analysis of measurements made on actual electrical equipment will almost certainly lack such input data.

Furthermore, the induced charge has been shown to be strongly dependent on the void orientation with respect to the applied field [113], and on the existence of a heterogenous bulk dielectric, see Section 7.3.2. 


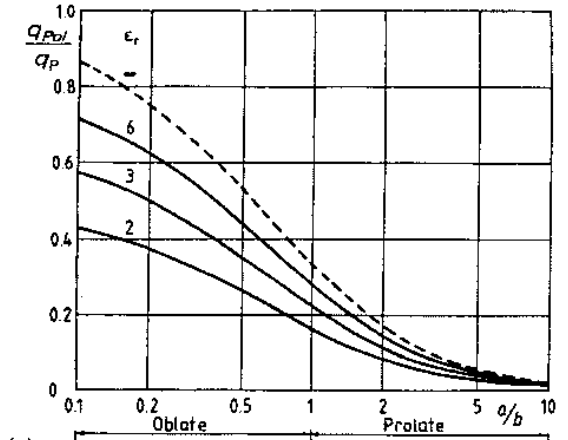

(a)

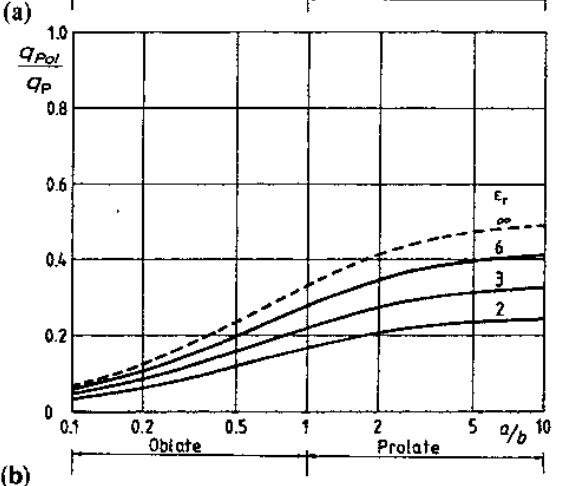

(b)

Figure 22. Variation of the induced charge component associated with changes in bulk dielectric polarization for spheroidal voids [76]. (a) $\vec{\nabla} \lambda_{0}$ parallel to void axis of rotation, (b) $\vec{\nabla} \lambda_{0}$ normal to void axis of rotation.

\subsubsection{BULK DIELECTRIC POLARIZATION}

The occurrence of partial discharges in gaseous voids leads not only to a charge being induced on the detecting electrode, but also to a change in the polarization of the bulk dielectric $[72,75]$. Mathematically, the final value of the Poissonian induced charge can be resolved into two components [76]

$$
q_{\mathrm{P}}=q_{\mu}+q_{\mathrm{pol}}
$$

where $q_{\mu}$ is the induced charge directly associated with the space charge in the void. For a dipole $\vec{\mu}$, this component is given by

$$
q_{\mu}=-\vec{\mu} \cdot \vec{\nabla} \phi
$$

The component $q_{\text {pol }}$ represents the induced charge related to the change in the dielectric polarization $(\delta \vec{P})$ due to the presence of this space charge. By combining (75), (81) and (82) we arrive at an expression for $q_{\text {pol: }}$ :

$$
q_{\mathrm{pol}}=-\vec{\mu} \cdot(\vec{\nabla} \lambda-\vec{\nabla} \phi)
$$

It is seen that the $q_{\mathrm{pol}}$ expression involves three independent vectors. Thus the general behavior of $q_{\text {pol }}$ is difficult to envisage. Hence initial studies of $q_{\mathrm{pol}}$ have concentrated on situations for which the three vectors were constrained to be parallel [114, 116-118].

For a homogeneous bulk dielectric, the magnitude of $q_{\text {pol }}$ is dependent on the void geometry, $\varepsilon_{r}$ of the bulk dielectric, and on the orientation of the void with respect to $\vec{\nabla} \lambda_{0}[76]$, see Figure 22 .
For a heterogeneous bulk dielectric, the behavior of $q_{\text {poi }}$ becomes more complex. For a two layer dielectric system, $q_{\text {pol }}$ increases when the void is located in the medium of lesser permittivity. In contrast, a decrease occurs when located in the medium of higher permittivity. This reduction can be of such a degree that the polarity of $q_{\mathrm{pol}}$ is reversed. This reversal occurs for permittivity ratios of $\sim 0.5$. In addition, when the void is in the dielectric of smaller physical extent, the greater is the relative increase/decrease in $q_{\mathrm{pol}}$. Full details of this $q_{\mathrm{pol}}$ behavior can be found in [114], while experimental evidence of the influence of the bulk dielectric polarization has been provided by Edin [115]:

For 3 or more dielectric layers, the magnitude of $q_{\mathrm{pol}}$ can be modified significantly in comparison to that associated with a 2-layer system. The nature of the change is essentially dependent on the relative permittivities of the various dielectrics [116].

Apart from the geometry of the bulk dielectric system, the void geometry also has a major influence on the magnitude and polarity of $q_{\text {pol }}$. This influence is dependent upon the prolateness/oblateness of the void geometry, and on the void orientation $[117,118]$.

For a fixed void location, $q_{\mu}$ is dependent only on $\vec{\mu}$ which, for comparative purposes, we can assume constant. Consequently variations in $q_{\text {pol }}$ will be reflected directly in the magnitude of the Poissonian induced charge $q_{\mathrm{P}}$, see (81). In certain conditions $q_{\mathrm{pol}}$ can change polarity, and as a consequence the magnitude of $q_{\mathrm{p}}$ is reduced even more, such that $q_{\mathrm{p}}<q_{\mu}$, see (81). However, under no circumstances can this lead to a change in the polarity of $q_{\mathrm{P}}[114]$.

Recently, hollow glass spheres have been used as a means of providing a reproducible source of partial discharges $[119,120]$. However, due to the dependency of $q_{\text {pol }}$ upon the complete dielectric system, the associated partial discharge transients are not uniquely determined by the hollow spherical geometry, even although there is a well-defined environment for partial discharge development, see [121].

\subsubsection{PARTIAL DISCHARGE DETECTION}

In previous Sections, the application of field theory to partial discharges has been illustrated. It is necessary, however, to introduce circuits and circuit theory when considering the question of partial discharge detection. The manner in which the two theories are interfaced will now be discussed.

The measuring system is composed of terminals and circuits. This system can be handled within the framework of circuit analysis. The dielectric system with its void and electrodes obeys the laws of fields. This system is thus not a part of the measuring system. The interconnection is established via the Poissonian/Laplacian induced charge relation, see Figure 14, such that, with respect to the external circuitry, the primary source for the partial discharge transient is the Laplacian induced charge. It is this component of the induced charge which can be manipulated by the observer.

The circuital aspects of partial discharge detection can be illustrated through the basic circuit shown in Figure 23. We assume that a partial discharge occurs in a void external to the electrode. With reference to the discharge transient, the governing equations for this circuit are [77]

$$
\begin{aligned}
q_{\mathrm{P}}(\dot{t}) & =\Delta Q(t)+C \Delta U(t) \\
\Delta U(t) & =Z i(t)
\end{aligned}
$$


and

$$
\Delta Q(t)=\int_{0}^{t} i\left(t^{\prime}\right) d t^{\prime}
$$

where $t^{\prime}$ is a dummy variable.

As we have a single electrode, (84) represents the simplest form of the induced charge expression [77]. In (84). $\Delta Q(t)$ denotes the charge transferred to the detecting electrode from the external source, while $\Delta U(t)$ represents the drop in potential of the detecting electrode, for which $C$ is the capacitance to ground. In (85) $Z$ is the impedance operator for the external circuit and $i(t)$ the transient current associated with the circuit. On selecting an appropriate $q_{\mathrm{p}}(t)$ variation, the circuit can be analyzed, see [77] for details. The results indicate that to achieve an accurate determination of the maximum value of the induced charge, one should record the voltage rather than the current transient.

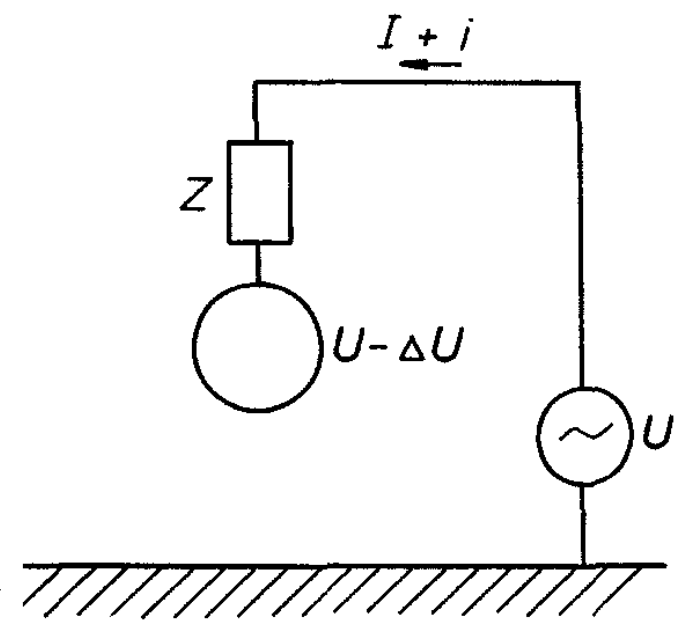

Figure 23. Basic detection system [77]. I power frequency current, $V$ power frequency voltage.

For a system of several electrodes, the expression for the induced charge becomes more complicated: The reason is that the partial discharge induces charge on all electrodes. Those electrodes not held at ground potential will undergo transient changes in potential. Due to the inherent partial capacitance associated with each pair of electrodes, such changes in potential will be registered at the detecting electrode. Thus for a system of $N$ electrodes, we have [75]

$$
q_{\mathrm{P} i}(t)=\Delta Q_{i}(t)+\sum_{j=0}^{N} C_{i j}\left[\Delta U_{i}(t)-\Delta U_{j}(t)\right]
$$

where $q_{P i}(t)$ denotes the Poissonian induced charge on the $i$ th electrode; $\Delta Q_{i}(t)$ denotes the charge transferred to the $i$ th electrode from the external source; $\Delta U_{i}$ and $\Delta U_{j}$ represent the drops in potential of the $i$ th and $j$ th electrodes from $U_{i}$ and $U_{j}$ to $\left(U_{i}-\Delta U_{i}\right)$ and $\left(U_{j}-\Delta U_{j}\right)$, respectively, while $C_{i j}$ is the partial capacitance between these electrodes. The subscript $j=0$ refers to ground and the parameter $t$ is time. A discussion of this expression for $N=2$ and $N=3$ may be found in [75].

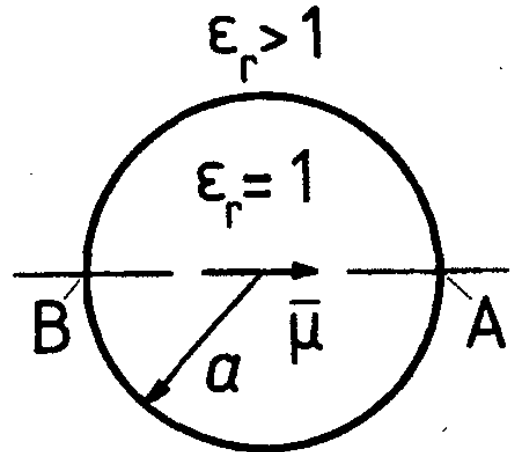

Figure 24. Model for field perturbation in a solid dielectric [122].

\subsection{FIELD ENHANCEMENT IN BULK DIELECTRIC}

\subsubsection{THEORETICAL BASIS}

The fact that a partial discharge induces a charge on the detecting electrode is a direct consequence of the partial discharge producing a perturbation of the electric field in the bulk dielectric. This field perturbation also brings about a change in the degree of polarization of the bulk dielectric. External to the void, the effect of the void wall charges can be considered equivalent to that of an electric dipole located within the void. This feature allows the nature of the field perturbation in the bulk dielectric to be assessed through a simple model.

Consider a point dipole of moment $\vec{\mu}$ located at the center of a spherical void in an extended dielectric, see Figure 24. From the field solution [122], the maximum field strength within the bulk dielectric is found to occur at $A$ and $B$. This field strength is given by

$$
\delta \vec{E}_{\mathrm{A}}=\delta \vec{E}_{\mathrm{B}}=\frac{2 h \vec{\mu}}{3 \varepsilon \Omega}
$$

External to the void, the applied field and the dipole field are additive. In Section 7.3.1, the dipole moment of a partial discharge in an ellipsoidal void is given as

$$
\vec{\mu}=\frac{K}{h} \varepsilon \Omega\left(\vec{E}_{\mathrm{i}}-\vec{E}_{l}\right)
$$

For a spherical void, $K=3$, and thus the partial discharge dipole moment for a spherical void is

$$
\vec{\mu}=\frac{3}{h} \varepsilon \Omega\left(\vec{E}_{\mathrm{i}}-\vec{E}_{l}\right)
$$

Consequently upon substituting (90) into (88) we obtain

$$
\delta \vec{E}_{\mathrm{A}}=\delta \vec{E}_{\mathrm{B}}=2\left(\vec{E}_{\mathrm{i}}-\vec{E}_{l}\right)
$$

To indicate the magnitude of this perturbation for a single discharge, reference is made to a spherical void of volume $1 \mathrm{~mm}^{3}$ filled with air at a pressure of $0.1 \mathrm{MPa}$. The streamer criterion yields the following value for $E_{\mathrm{i}}$, viz.

$$
\begin{array}{ll}
E_{\mathrm{i}}=4.3 & \mathrm{kV} / \mathrm{mm} \\
E_{l}=2.4 \quad \mathrm{kV} / \mathrm{mm}
\end{array}
$$

Hence in the proximity of the void, (91) indicates that a single discharge could generate a field distortion within the solid dielectric of $\sim 4 \mathrm{kV} / \mathrm{mm}$. This value can quite easily exceed the applied field at $\mathrm{A}$ and $B$ by a factor 2 . 
It must be realized however that just as $\vec{\mu}$ is dependent on the specific assumptions made relating to the partial discharge development and the corresponding $\sigma$ distribution, so too is the field perturbation, see [123].

\subsubsection{PRACTICAL ASPECTS}

In practice for an ac voltage, the applied field strength in the void will be much greater than the inception field strength for a partial discharge. This situation will result in several partial discharges per cycle. If the surface conductivity of the void wall is taken as zero, then the partial discharge wall-charge will remain in situ and the field distortion remains at a constant level until the occurrence of the next discharge. If this discharge occurs in the same direction, then the field distortion will be further increased. Consequently, a unidirectional sequence of partial discharges results in a cumulative field distortion within the solid dielectric in the vicinity of the void. For the spherical void under consideration, this distortion can be expressed as [124]

$$
\delta \vec{E}_{\max }=N\left(\vec{E}_{\mathrm{i}}-\vec{E}_{l}\right)
$$

where $N$ is the total number of either positive or negative discharges per cycle.

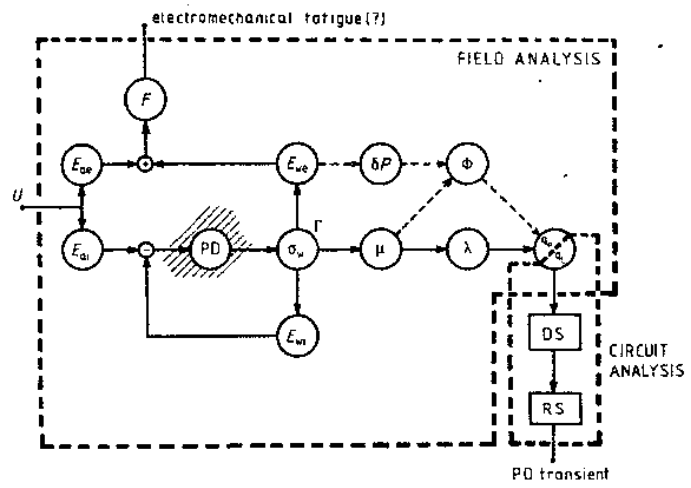

Figure 25. PD transients: Theoretical framework. PD partial discharge; DS detecting system; $\mathrm{RS}$ recording system; $U$ applied voltage; $\lambda$ the $\lambda$ function; $\phi$ the $\phi$ function; $q_{\mathrm{P}}$ Poissonian induced charge; $q_{\mathrm{L}}$ Laplacian induced charge. With reference to the void: $\sigma_{\mathrm{w}}$ void -wall charge; $E_{\mathrm{ai}}$ internal applied field; $E_{\mathrm{wi}}$ internal wall charge field; $E_{\text {ae }}$ external applied field; $E_{\text {we }}$ external wall charge field; $F$ electrical force generated by $\left(E_{\text {ae }}+E_{\text {we }}\right) ; \delta P$ change in bulk dielectric polarization due to $E_{\text {we }} ; \Gamma$ surface conductivity of the void wall; $\mu$ dipole moment of $\sigma_{\mathrm{w}}$.

In contrast to the very short time duration of each partial discharge current pulse, in the nanosecond range, the maximum value of the induced charge, or field distortion, associated with several partial discharges will persist essentially as a quasi-dc signal: i.e. with a duration of $\sim 5 \mathrm{~ms}$ for an applied voltage at power frequency.

The significance of the partial discharge field enhancement is that the electrostatic forces $F$ are proportional to the square of the electric field strength $E$, i.e. $F \propto E^{2}$, and thus such field enhancement could lead to significant force levels within the solid dielectric. This electromechanical stressing may be the catalyst which promotes electrical treeing.
If $E_{\mathrm{v}}$ represents the net field within the void, then the above analysis can be extended by considering the limiting condition of $E_{\mathrm{v}} \rightarrow$ 0 following several partial discharges [125]. For this condition, the maximum possible field strength generated within the solid dielectric bounding a void can be deduced as a result of the partial discharge activity in this void. In turn, this field value allows the corresponding maximum possible dipole moment to be estimated, see [125]. With respect to test procedures; this dipole moment enables a meaningful, maximum level of induced charge to be specified for which the integrity of the solid dielectric is not compromised.

\subsection{DISCUSSION}

A physically valid theory of partial discharge transients has been developed using a field-theoretical approach. Through this approach a framework has evolved, which links in a logical manner the different topics discussed in this Section, see Figure 25. The result is a cohesive, self-consistent view of the theoretical aspects of partial discharge transients.

Beginning at the left-hand side of this Figure, we have the applied voltage $U$ generating an electric field external to the void and also an internal field, viz. $E_{\mathrm{ae}}$ and $E_{\mathrm{ai}}$, respectively. When the internal field is larger than the inception field strength for the void gas, a partial discharge can develop. As a consequence of this discharge, charge separation occurs within the void. Subjected to the applied field, these charges accumulate at the void wall, and by virtue of their polarities generate a field $E_{\text {wi }}$, which opposes the applied field $E_{\text {ai }}$ within the void. When the net field in the void $\left(E_{\mathrm{ai}}-E_{\mathrm{wi}}\right)$ has been reduced sufficiently, i.e. less than the limiting field strength for ionization growth, the partial discharge will be quenched.

With respect to the detecting electrode, the effect of the void wall charges is equivalent to that of an electric dipole of moment $\vec{\mu}$ located within the void. Through the $\lambda$ function, the Poissonian induced charge $q_{\mathrm{P}}$ arising from $\vec{\mu}$ can be evaluated. The associated Laplacian induced charge $q_{\mathrm{L}}$ serves as the source for the detection and recording systems. Consequently $q_{\mathrm{L}}$ is the essential interfacing parameter between the field and circuit domains.

The surface conductivity $\Gamma$ of the void wall affects the persistence of the wall charge [126]. If $\Gamma=0$, then the wall charge will remain in situ. The occurrence of the next discharge will, depending on its polarity, either augment or reduce the magnitude of the wall charge.

External to the void, the wall charge generates a field $E_{\mathrm{we}}$, which augments the applied field $E_{\text {ae. }}$. Because electrostatic forces $F$ are proportional to $E^{2}$, the field enhancement arising from partial discharge activity could promote electromechanical fatigue of the solid dielectric.

$E_{\text {we }}$ also perturbs the polarization of the solid dielectric. The induced charge associated with the change in polarization, $\delta \vec{P}$, can be evaluated by using the $\phi$ function.

The major components of the partial discharge framework are undoubtedly in place. However there is still much detail to be uncovered; e.g. the response of different detecting systems, the influence of surface conductivity, especially with reference to 'DC voids', the field enhancement in the solid dielectric, and the associated electromechanical effects. 
- In conclusion, a comparison of Figure 19 with Figure 25 amply demonstrates the power of a field-theoretical approach.

\section{CONCLUSIONS}

From the review of progress achieved over the past 30 years in different aspects of electrical insulation, it has been demonstrated clearly that a field-theoretical approach is very fruitful. An added benefit from using this approach is that initial results lead frequently to unforeseen developments; e.g. the inherent non-uniformity of uniform field gaps and the precise requirements for valid Paschen curve measurements, surface roughness modeling and the figure of merit for strongly electronegative gases/gas-mixtures, partial discharge transients and the significance of changes in the bulk dielectric polarization. Such developments should serve to encourage younger research workers to adopt a field-theoretical approach from the onset of any project.

\section{ACKNOWLEDGMENT}

The author wishes to acknowledge his debt to a very synergistic cooperation with his former colleagues A. Pedersen, P. W. Karlsson, and last but not least, G. C. Crichton. The author also wishes to thank J. Berril for the production and reproduction of all the Figures, in particular those from the $1970 \mathrm{~s}$

\section{APPENDIX}

\subsection{THE STREAMER CRITERION}

For non-uniform fields, the discharge onset conditions for a gaseous dielectric can be evaluated using the streamer criterion. Although this criterion is based upon considerations of electron avalanche development, see [12], it is essentially an empirical criterion, which is expressed as

$$
\int_{0}^{z_{0}} \bar{\alpha}(z) d z=K
$$

The parameter $\bar{\alpha}$ is the effective coefficient of ionization for the gas in question, and $z_{0}$ is the critical avalanche length. This length is the distance from the highly stressed electrode along the field line in question to the point beyond which ionization growth ceases, i.e. to the point at which $\bar{\alpha}=0$. Although various interpretations have been placed on $K$, it is essentially a dimensionless parameter. A method of evaluating this parameter is described in Section 2.3.

\subsection{UNIT CONVERSION FACTORS}

For gas pressure: $p$

$$
1 \mathrm{~mm} \mathrm{Hg}=1.333 \times 10^{-3} \text { bar; } 1 \text { bar }=0.1 \mathrm{MPa}
$$

For parameter $p d$ :

$$
1 \mathrm{~mm} \mathrm{Hg} \mathrm{cm}=1.333 \times 10^{-2} \text { bar } \mathrm{mm}
$$

For parameter $(E / p)$ :

$$
\begin{gathered}
1 \frac{\mathrm{V} \mathrm{cm}^{-1}}{\mathrm{~mm} \mathrm{Hg}}=7.501 \times 10^{-2} \frac{\mathrm{kV} \mathrm{mm}^{-1}}{\mathrm{bar}} \\
\text { REFERENCES }
\end{gathered}
$$

[1] O. D. Kellogg, Foundations of Potential Theory, J. Springer, Berlin, 1929; Dover, New York, 1954.
[2] A. Pedersen, P. W. Karlsson and J. Lebeda, "The Effect of Field Nonuniformity and Asymmetry on Ionization Current Growth Measurements in Sulfur Hexafluoride", IEEE Trans. Power Appar. \& Syst., Vol. PAS-90, pp. 2175-2180, 1971.

[3] P. W. Karlsson and A. Pedersen, "Inherent Limitations in Uniform Field Discharge Data for $\mathrm{SF}_{6}{ }^{\prime \prime}$, IEEE Trans. Power Appar. \& Syst., Vol. PAS-91, pp. 1597-1601, 1972.

[4] T. Munk Nielsen and A. Pedersen, "Edge Breakdown between Uniform Field Electrodes in $\mathrm{SF}_{6}{ }^{\prime \prime}$, 2nd Intemational Conference on Gas Discharges, London 1972. IEE Conference Publication No.90, pp. 323-325, 1972.

[5] A. Pedersen, P. W. Karlsson, E. Bregnsbo and T. Munk Nielsen, "Anomalous Breakdown in Uniform Field Gaps in $\mathrm{SF}_{6}{ }^{\prime}$, IEEE Trans. Power Appar. \& Syst., Vol. PAS-93, pp. 1820-1826, 1974.

[6] H. A. Boyd and G. C. Crichton, "Measurement of Ionisation and Aftachment Coefficients in $\mathrm{SF}_{6}{ }^{\prime \prime}$, Proc. IEE, Vol. 118, pp. 1872-1877, 1971

[7] E. Kuffel and W. S. Zaengl, High-Voltage Engineering: Fundamentals, Pergamon, Oxford, 1984.

[8] E. Kuffel, W. S. Zaengl and J. Kuffel, High Voltage Engineering: Fundamentals, Second edition, Newnes, Oxford, 2000

[9] H. A. Boyd and G. C. Crichton,"Uniform-Field Breakdown-Voltage Measurements in Sulphur Hexafluoride", Proc. IEE, Vol. 119, pp. 275-276, 1972.

[10] T. Leardini and P. Peiser, "Progress Report of Study Committee No.15", Electra, No.27, pp. 5-13, 1973.

[11] T. W. Dakin, G. Luxa, G. Oppermann, J. Vigreux, G. Wind, H. Winkelnkemper, "Breakdown of Gases in Uniform Fields, Paschen Curves for Nitrogen, Air and Sulfur Hexafluoride", Electra, No.32, pp. 61-82, 1974.

[12] A. Pedersen, I. W. McAllister, G. C. Crichton and S. Vibholm, "Formulation of the Streamer Breakdown Criterion and its Application to Strongly Electronegative Gases and Gas Mixtures", Archiv für Elektrotechnik, Vol. 67, pp. 395-402, 1984

[13] I. W. McAllister and A. Pedersen,"Corona-Onset Field-Strength Calculations and the Equivalent Radius Concept", Archiv für Elektrotechnik, Vol, 64, pp. 43-48, 1981.

[14] A. Pedersen,"On the Electrical Breakdown of Gaseous Dielectrics: An Engineering Approach", IEEE Trans. Elect. Insul., Vol. 24, pp. 721-739, 1989.

[15] J. Berril, J. M. Christensen and A. Pedersen,"Measurement of the Figure of Merit Related to the Effect of Electrode Surface Defects on Breakdown for Strongly Electronegative Gases or Gas Mixtures", Seventh International Conference on Gas Discharges and Their Applications, London 1982. Peter Peregrinus, London, pp. 266 268,1982 .

[16] J. Berril, J. M. Christensen and I. W. McAllister,"Measurement of the Figure of Merit $M$ for for $1-\mathrm{C}_{3} \mathrm{~F}_{6}, 1,2-\mathrm{C}_{2} \mathrm{Cl}_{2} \mathrm{~F}_{4}$ and $\mathrm{C}_{2} \mathrm{ClF}_{5}$ ", Eighth International Conference on Gas Discharges \& Their Applications, Oxford, pp. 239-242, 1985.

[17] J. Berril, J. M. Christensen and I. W. McAllister,"Measurement of the Figure of Merit $M$ for Several Strongly Electronegative Gases", Conference Record of the 1986 IEEE International Symposium on Electrical Insulation, Washington D.C. IEEE Publication 86CH2196-4-DEL, pp. 251-257, 1986.

[18] J. Berril, J. M. Christensen and I. W. McAllister, "Measurement of the Figure of Merit $M$ for Several Perfluocarbon Gases", in L. G. Christophorou and D. W. Bouldin (eds.), Gaseous Dielectrics V, Pergamon Press, New York, pp. 304-310, 1987

[19] J. Berril, J.M. Christensen and I. W. McAllister, "Measurement of the Figure of Merit $M$ for $\mathrm{CBrClF}_{2}, \mathrm{CBr}_{2} \mathrm{~F}_{2}, \mathrm{CBrF}_{3}$ and $\mathrm{CClF}_{3}{ }^{\prime \prime}$, Fifth International Symposium on High Voltage Engineering, Braunschweig, Paper 15.16, 1987.

[20] J. Berril and I. W. McAllister,"Measurement of the Figure of Merił $M$ for Several $\mathrm{N}_{2}$-based Binary Gas Mixtures", Sixth International Symposium on High Voltage Engineering, New Orteans, Paper 32.08, 1989.

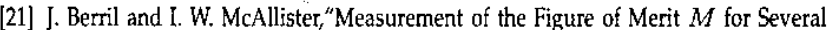
$\mathrm{SF}_{6}$-based Binary Gas Mixtures", in L. G. Christophorou and I. Sauers (eds.), Gaseous Dielectrics VI, Plenum Press; New York, pp. 193-199, 1991.

[22] G. Berger and B. Senouci, "'The Role of Impurities on the Deviation from Paschen's Law of $\mathrm{SF}_{6}{ }^{\prime \prime}$; J. Phys. D: Appl. Phys., Vol. 19, pp. 2337-2342, 1986.

[23] I. W. McAllister and G. C. Crichton, "The Concept of Paschen's Law with Reference to $\mathrm{SF}_{6}{ }^{\prime \prime}$, J. Phys. D: Appl. Phys., Vol. 20, pp. 1537-1539, 1987.

[24] I. W. McAllister,"Illusory Paschen Curves Associated with Strongly Electronegative Gases", IEEE Trans. Elect. Insul., Vol. 26, pp. 391-397, 1991. 
[25] B. H. Crichton and D. J. Tedford, "The Application of Low-Pressure Experimental Data to the Calculation of Electrical Discharge Thresholds in Compressed Gases", j. Phys. D: Appl. Phys., Vol. 9, pp. 1079-1083, 1976.

[26] H. Steinbigler, "Anfangsfeldstärken und Ausnutzungsfaktoren rotations-symmetrischer Elektrodenanordnungen in Luft", Diss. Technischen Hochschule München 1969.

[27] H. Steinbigler, "Digitale Berechnung elektrischer Felder", ETZ-A, Vol. 90, pp. 663$666,1969$.

[28] H. Singer,"Das elektrische Feld von Gitterelektroden", ETZ-A, Vol. 90, pp. 682-686, 1969.

[29] P. Weiss, "Berechnung von Zweistoffdielektrika", ETZ-A, Vol. 90, pp. 693-694, 1969.

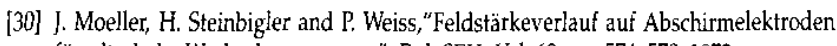
für ultrahohe Wechselspannungen", Bul. SEV., Vol. 63, pp. 574-578, 1972.

[31] H. Singer," Das elektrische Feld von Polycon-Elektroden”, Bull. SEV., Vol. 63, pp. $579-583,1972$.

[32] P. Weiss, "Feldstärkeeffekte bei Zweistoffdielektrika", Bull. SEV., Vol. 63, pp. 584 $588,1972$.

[33] H. Singer, H. Steinbigler and P. Weiss, "A Charge Simulation Method for the Calculation of High Voltage Fields", IEEE Trans. Power Appar. \& Syst., Vol. PAS-93, pp. 1660-1668, 1974.

[34] I. W. McAllister and A. Pedersen,"Green's Differential Equation and Electrostatic Fields", J. Phys. D: Appl. Phys., Vol. 21, pp. 1823-1825, 1988. Erratum, ibid. Vol. 22, p.473, 1989.

[35] A. J. Davies, K. E. Donne and C. J. Hill,"Edge Breakdown in Electronegative Gases between Uniform Field Electrodes", 4th International Conference on Gas Discharges, Swansea 1976. IEE Conference Publication No.143, pp. 176-179, 1976.

[36] A. Pedersen and E. Bregnsbo,"Estimation of Breakdown Voltages in Compressed $\mathrm{SF}_{6}{ }^{\prime \prime}$, Internationales Symposium Hochspannungstechnik, Zürich, pp. 432-436, 1975 .

[37] W. Mosch and W. Hauschild, "Möglichkeiten zur Berechnung der Durchschlagspannung schwach inhomogener Anordnungen in $\mathrm{SF}_{6}{ }^{\prime \prime}$, Elektrie, Vol. 28, pp. 152156,1974 .

[38] W. Mosch and W. Hauschild, "Calculating the Breakdown Voltage in $\mathrm{SF}_{6}$ in a System of Coaxial Cylinders", Elektrichestvo, No.5, pp. 50-54, 1974, or Electric Technology USSR, No.2, pp. 71-81, 1974.

[39] A. L. Vilenchuk, "Effect of the Material and Surface Finish of Coaxial Electrodes on the Electric Strength of $\mathrm{SF}_{6}{ }^{\prime \prime}$, Elektrichestvo, No.11, pp. 70-72, 1970.

[40] C. Ikeda and B. Yoda,"Effect of Electrode Surface Conditions and Dust Particles on the Electrical Breakdown of $\mathrm{SF}_{6} \mathrm{Gas}^{\prime \prime}$, Electrical Engineering in Japan, Vol. 91, No.5, pp. 67-74, 1971 .

[41] P. Hogg, W. Schmidt and H. Strasser, "Dimensioning of $\mathrm{SF}_{6}$ Metalclad Switchgear to Ensure High Reliability", CIGRE Paper 23-10, pp. 1-12, 1972.

[42] T. Nitta, N. Yamada and Y. Fujiwara, "Area Effect of Electrical Breakdown in Compressed SF6", IEEE Trans. Power Appar. \& Syst., Vol. PAS-93, pp. 623-629, 1974.

[43] A. Pedersen, "Limitations of Breakdown Voltages in $\mathrm{SF}_{6}$ Caused by Electrode Surface Roughness", 1974 Annual Report, Conference on Electrical Insulation and Dielectric Phenomena, Downingtown, PA 1974. National Academy of Sciences, Washington, D.C., pp. 457-464, 1975.

[44] R. Le Ny, J. Charrier and A. Boulloud, "Simple Models in Room Air Simulating the Influence of Conducting Microrugosity of the Electrodes on the Breakdown Voltage in Compressed Gases", 4th International Conference on Gas Discharges, Swansea, 1976. IEE Conference Publication No.143, pp. 121-124, 1976.

[45] R. Le Ny, "The Electrostatic Problem of a Portion of Sphere Protruding from a Plane Electrode in an Electric Field", J. Phys. A: Math. \& Gen., Vol. 14, pp. 945-955, 1981.

[46] I. W. McAilister, "On the Electrostatics of Spherical Protrusions", IEE Proc., Vol. 133, part A, pp. 84-88, 1986 .

[47] J. Charrier, R. Le Ny and A. Boulioud, "Influence of a Spherical Protrusion on one of the Electrodes on the Breakdown Voltage in Room Air", Third International Conference on Gas Discharges, London 1974. IEE Conference Publication No.118, pp. $394-398,1974$.
[48] S. Berger, "Onset or Breakdown Voltage Reduction by Electrode Surface Roughness in Air and $\mathrm{SF}_{6}{ }^{\prime}$, IEEE Trans. Power Appar. \& Syst., Vol. PAS-95, pp. 1073-1079, 1976.

[49] V. G. Agapov and D. V. Razevig, "Breakdown Voltages of Gaseous Gaps at High Pressures", Elektrichestvo, No.5, pp. 32-34, 1972 or Electric Technology USSR, Vol. 2, pp. 76-83, 1972.

[50] V. A. Avrutskii, G. M. Goncharenko and E. N. Prokhorov, "Effect of Electrode Roughness on the Electrical Strength of Compressed Gases", Sov. Phys., Tech. Phys., Vol. 18, pp. 386-388, 1973 .

[51] V. G. Agapov and M. V. Sokolova,"The Influence of Elec:rode Surface Microwhiskers on a Breakdown Voltage of a Pressurized Gas", Internationales Symposium Hochspannungstechnik, Zürich, pp. 395-398, 1975.

[52] 1. W. McAllister, "A Multiple Protrusion Model for Surface Roughness Effects in Compressed SF,", ETZ-A, Vol. 99, pp. 283-284, 1978.

[53] I. W. McAllister,"On the Concept of Electrode Surface Roughnes; with Reference to Discharge Phenomena in Strongly Electronegative Gases", IEEE Trans. Elect. Insul., Vol. EI-21, pp. 659-662, 1986.

[54] I. W. McAllister and E. Bregnsbo, ${ }^{a}$ Electrode Surface Modelling and the Prediction of Breakdown in Compressed $\mathrm{SF}_{6}{ }^{\prime}$, Seventh International Conference on Gas Discharges and Their Applications, London 1982. Peter Peregrinus Ltd., London, pp. 262-265, 1982.

[55] I. W. McAllister, "The Influence of Electrode Macroscopic Curvature upon Surface Roughness Effects in Compressed $\mathrm{SF}_{6}{ }^{\prime \prime}$, Archiv für Elektrotechnik, Vol. 62, pp. 43$49,1980$.

[56] A. Pedersen, "The Effect of Surface Roughness on Breakdown in $\mathrm{SF}_{6}$ ", IEEE Trans. Power Appar. \& Syst., Vol. PAS-94, pp. 1749-1754, 1975.

[57] G. Oppermann, "Die Paschen-Kurve und die Gültigkeit des Paschen Gesetzes für Schwefelhexafluorid", Internationales Symposium Hochspannungstechnik, München, pp. 378-385, 1972.

[58] A. Pedersen, "Anomalous Phenomena Related to Paschen's Law for $\mathrm{SF}_{6}{ }^{\prime}$, Nordisk Symposium om Isolationsteknik (NORD-IS 74), Copenhagen, Paper 24, 1974.

[59] W. Zaengl and R. Baumgartner,"Zur Ursache der Abweichungen vom Paschengesetz in SF ${ }^{\prime \prime}$, ETZ-A, Vol. 96, pp. 510-514, 1975.

[60] O. Farish, O. E. Ibrahim and B. H. Crichton,"Effect of Electrode Surface Roughness on Breakdown in Nitrogen/SF 6 Mixtures", Proc. IEE, Vol. 123, pp. 1047-1050, 1976.

[61] S. Berger, ${ }^{\prime \prime}$ Influence of a Single Electrode Surface Protrusion on the Orset Voltage in Air and $\mathrm{SF}_{6}{ }^{\prime \prime}$, IEEE PES Summer Power Meeting, San Francisco, Paper No.A 75 494-5, 7 pp. , 1975.

[62] S. Berger, "Einfluss von definierten Elektrodenoberflächenrauhigkeiten auf die Einsatzspannung in Luft und $\mathrm{SF}_{6}$ ", Internationales Symposium Hochspannungstechnik, Zürich, pp. 399-404, 1975.

[63] R. Baumgartner,"Versuche zur Ursache der Abweichungen von Paschengesetz in $\mathrm{SF}_{6}{ }^{\prime \prime}$, ETZ-A, Vol. 97, pp. 177-178, 1976.

[64] G. Biasiutti, C. Ammann, E. Englet and W. Zaengl,"Electric Strength of Hexafluoropropylene $\left(\mathrm{C}_{3} \mathrm{~F}_{6}\right)$ and its Mixtures with $\mathrm{SF}_{6}$ at Practical Pressures", Fourth International Symposium on High Voltage Engineering, Athens, Paper No.33.02, 1983.

[65] American National Standard, Surface Textures (Surface Roughness, Waviness and Lay), ANSI B46.1-1978, The American Society of Mechanical Engineers NY 1978.

[66] A. Pedersen,"Evaluation of the Effect of Surface Defects on Breakdown in Strongly Electronegative Gases or Gas Mixtures", in L. G. Christophorou (Ed.), Gaseous Dielectrics II, Pergamon Press, New York, pp. 201-209, 1980.

[67] I. W. McAllister,"On the Choice of a Test Gap for Strongly Electronegative Gases and Gas Mixtures", in L. G. Christophorou and M. O. Pace (eds.), Gaseous Dielectrics $I V$, Pergamon Press, New York, pp. 195-203, 1984.

[68] G. C. Crichton and S. Vibholm, "Discharge Onset Voltage Prediction for a GasInsulated System via the Figure-of-Merit Concept", IEEE Trans. Elec. Insul, Vol. 22, pp. $819-823,1987$.

[69] G. C. Crichton, I. Drimal, I. W. McAllister and S. Vibholm, "Discharge Onset Prediction on 1,2- $\mathrm{C}_{2} \mathrm{Cl}_{2} \mathrm{~F}_{4} / \mathrm{SF}_{6}$ Mixtures via the Figure-of-Merit Concept", IX International Conference on Gas Discharges and Their Applications, Venezia, pp. 307-310, 1988 .

[70] I. W. McAllister and G. C. Crichton,"An Experimental Methoci to Determine the Electrostatic Field Enhancement Factor of a Practical Conductor Surface", IEEE Trans. Elect. Insul., Vol. 24, pp. 325-333, 1989. Erratum: ibid., Vol. 24, p.572, 1989.

[71] I. W. McAllister and S. Vibholm,"The Field Enhancement Factor of Sand-Blasted Electrodes", Conference Record of the 1992 IEEE International Symposium on Electrical Insulation, Baltimore. IEEE Publication 92CH3150-0, pp. 307-309, 1992. 
[72] A. Pedersen, "Current Pulses Generated by Discharges in Voids in Solid Dielectrics. A Field Theoretical Approach", Conference Record of the 1986 IEEE International Symposium on Electrical Insulation, Washington, DC. IEEE Publication 86CH21964-DEI, pp. 112-114, 1986.

[73] A. Pedersen,"On the Electrostatics of Probe Measurements of Surface Charge Densities", in L. G. Christophorou and D. W. Bouldin (eds.), Gaseous Dielectrics V, Pergamon Press New York, pp. 235-241, 1987.

[74] A. Pedersen, "Partial Discharges in Voids in Solid Dielectrics. An Alternative Ap-

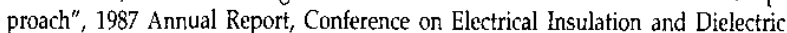
Phenomena, Gaithersburg, MD. IEEE Publication 87CH2462-0, pp. 58-64, 1987.

[75] A. Pedersen, G. C. Crichton and I. W. McAllister,"The Theory and Measurement of Partial Discharge Transients" ${ }^{\prime \prime}$ IEEE Trans. Elect. Insul,, Vol. 26, pp. 487-497, 1991.

[76] A. Pedersen, G. C. Crichton and I. W. McAllister, ${ }^{, T}$ The Functional Relation between Partial Discharges and Induced Charge", IEEE Trans. Diel. Elect. Insul., Vol. 2, pp. 535-543, 1995

[77] A. Pedersen, G. C. Crichton and I. W. McAllister, ${ }^{\prime}$ Partial Discharge Detection: Theoretical and Practical Aspects", IEE Proc., Science, Measurement \& Technology, Vol. 142 , pp. 29-36, 1995.

[78] D. K. Davies,"The Examination of the Electrical Properties of Insulators by Surface Charge Measurement", J. Sci. Instrum., Vol. 44, pp. 521-524, 1967.

[79] T. R. Foord,"Measurement of the Distribution of Surface Electric Charge by Use of a Capactive Probe", J. Phys. E, Vol. 2, pp. 411-413, 1969,

[80] P. Connolly and O. Farish, "Surface Charge Measurement in Air and $\mathrm{SF}_{6}$, in L. G. Christophorou and M. O. Pace (eds.), Gaseous Dielectrics IV, Pergamon Press, New York, pp. 405-413, 1984.

[81] N. C. Jaitly and T. S. Sudarshan, "In-situ Insulator Surface Charge Measurements in Dielectric Bridged Vacuum Gaps Using an Electrostatic Probe", IEEE Trans. Elect. Insul., Vol. 23, pp. 261-273, 1988.

[82] T. Jing, P. H. F. Morshuis and F. H. Kreuger, "The Calibration of a Capactive Probe for Surface Charge Measurements", Archiv für Elektrotechrik, Vol. 73, pp. 337-341, 1990

[83] H. Ootera and K. Nakanishi,"Analytical-Method for Evaluating Surface Charge Distribution on a Dielectric from Capacitive Probe Measurement, Application to a Cone-Type Spacer in $\pm 500 \mathrm{kV}$ DC-GIS", IEEE Trans. Power Delivery, Vol. 3 , pp. 165-172, 1988. (presented at the IEEE-PES Summer Meeting, Mexico City, 1986)

[84] M. Yashima, H. Fujinami and T. Takuma," Measurement of Accumulated Charge on Dielectric Surfaces with an Electrostatic Probe", in L. G. Christophorou and D. W. Bouldin (eds.), Gaseous Dielectrics V, Pergamon Press, New York, pp. 242-248, 1987.

[85] C. E. Sudhakar and K. D. Srivastava,"Electric Field Computation from Probe Measurements of Charge on Spacers Subjected to Impulse Voltages", Fifth International Symposium on High Voltage Engineering, Braunschweig, Paper 33.14, 1987.

[86] H. Specht, "Oberflächenladungen bei rotationssymmetrischen Isolierstoffkörpern", ETZ-A, Vol. 97, pp. 474-476, 1976.

[87] T. O. Rerup, G. C. Crichton and I. W. McAllister, "Using the $\lambda$ Function to Evaluate Probe Measurements of Charged Dielectric Surfaces", IEEE Trans. Diel. Elect. Insul., Vol. 3, pp. 770-777, 1996.

[88] T. Takuma, M. Yashima and T. Kawamoto,"Principle of Surface Charge Measurement for Thick Insulating Specimens", IEEE Trans. Diel. Elect. Insul, Vol. 5, pp. 497-504, 1998

[89] T. Takuma and 5. Hamada, "Analysis of Measuring Methods for Accumulated Surface Charge", Elektrie, Vol. 53, pp. 397-400, 1999.

[90] T. O. Rerup, G. C. Crichton and I. W. McAlister, "The Response of Electrostatic Probes via the $\lambda$ Function", Conference Record of the 1994 IEEE International Symposium on Electrical Insulation, Pittsburgh PA. IEEE Publication 94CH3445-4, pp. $82-88,1994$

[91] T. O. Rerup, G. C. Crichton and I. W. McAllister, "The $\lambda$ Function and the Response of Electrostatic Probes", Nordic Insulation Symposium (NORD-IS 94), Vaasa, Finland, Paper No.7.7, pp. 343-351, 1994.

[92] T. O. Rerup, G. C. Crichton and I. W. McAllister, "Response of an Electrostatic Probe for a Right Cylindrical Spacer", 1994 Annual Report, Conference on Electrical lnsulation and Dielectric Phenomena, Arlington TX. IEEE Publication 94CH3456-1, pp. $167-176,1994$
[93] T. Johansson, G. C. Crichton and I. W. McAllister,"Influence of Probe Geometry on the Response of an Electrostatic Probe", 1999 Annual Report, Conference on Electrical Insulation and Dielectric Phenomena, Austin TX. IEEE Publication 99CH36319, pp. 137-141, 1999

[94] T. Johansson, G. C. Crichton and I. W. McAllister,"Measurement of Surface Charge Densities Associated with Surface Discharges", XIII International Conference on Gas Discharges and Their Applications, Glasgow, pp. 892-895, 2000.

[95] T. Johansson and I. W. McAllister,"Response of Electrostatic Probes to Non-uniform Charge Distributions", Nordic Insulation Symposium (NORD-IS 01), Stockholm, pp. 271-279, 2001.

[96] T. Johansson and I. W. McAllister, ${ }^{\prime}$ Response of Electrostatic Probes to Eccentric Charge Distributions", 2001 Annual Report, Conference on Electrical Insulation and Dielectric Phenomena, Kitchener ON, IEEE Publication 01CH37225, pp. 652-655, 2001.

[97] M. Endo, N. Noro and T. Sakakibara," Field Strength of Surface Discharge initiation (II)", Eleventh International Conference of Gas Discharges and Their Applications, Tokyo, pp. II/118-II/121, 1995.

[98] M. Endo, N. Harigaya, H.Suzuki and T. Sakakibara, "Field Strength of Surface Discharge Initiation (III)", Twelfth International Conference on Gas Discharges and Their Applications, Greifswald, pp. I/260-I/263, 1997.

[99] O. Yamamoto, T. Takuma and Y. Tanabe,"Real-Time Observation of Surface Charging on a Cylindrical Insulator in Vacuum", IEEE Trans. Diel. Elect. Insul., Vol. 5, pp. $961-965,1998$.

[100] O. Yamamoto, T. Takuma, Y. Kakehashi, S. Ikoma and T. Shioiri, "Delay Characteristics of Surface Charging on a Cylindrical Insulator in Vacuum", IEEE Trans. Diel. Elect. Insul., Vol. 7, pp. 812-817, 2000.

[101] J. M. Meek and ]. D. Craggs (eds.), Electrical Brenkdown of Gases, Wiley, Chichester, 1978.

[102] I. W. McAllister, "Electric Field Theory and the Fallacy of Void Capacitance", IEEE Trans. Elect. Insul, Vol. 26, pp. 458-459, 1991.

[103] R. Bartnikas, "A Commentary on Partial Discharge Measurement and Detection", IEEE Trans. Electr. Insul., Vol. E1-22, pp. 629-653, 1987.

[104] R. Bartnikas, "Partial Discharge Diagnostics: A Utility Perspective", in L. G. Christophorou and D. R. James (eds.), Gaseous Dielectrics VII, Plenum Press, New York, pp. 209-222, 1994.

[105] D. P. Agoris and N. D. Hatziargyriou, "Approach to Partial Discharge Development in Closely Coupled Cavities Embedded in Solid Dielectrics by Lumped Capacitance

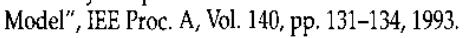

[106] D. P. Agotis and N. D. Hatziargyriou,"A Modelistic Consideration of the Interaction Between Discharges in Cavities of Solid Dielectric", 8th International Symposium of High Voltage Engineering, Yokohama, Paper 63.12, 1993.

[107] M. G. Danikas, "Discharge Studies in Solid Insulation Voids", 1990 Annual Report, Conference on Electrical Insulation and Dielectric Phenomena, Pocono Manor, PA. IEEE Publication 90CH2919-9, pp. 249-254, 1990.

[108] M. G. Danikas, "Some Further Comments on the Fast Measurements of Partial Discharges in Polyethylene Voids", 20th Electrical Electronics Insulation Conference, Boston. IEEE Publication 91CH2991-8, pp. 220-224, 1991.

[109] M. G. Danikas and A. M. Bruning, "Comparison of Several Theoretical Sub-corona to Corona Transition Relations with Recent Experimental Results", Conference Record of the 1992 IEEE International Symposium on Electrical Insulation, Baltimore, MD. IEEE Publication 92CH3150-0, pp. 383-388, 1992.

[110] J. A. Stratton, Electromagnetic Theory, McGraw-Hill, New York, 1941

[111] I. W. McAllister, "The Dipole Moment of a Wall-charged Void in a Bulk Dielectric", 1993 Annual Report, Conference on Electrical Insulation and Dielectric Phenomena, Pocono Manor PA. IEEE Publication 93CH3269-8, pp. 468-473, 1993.

[112] G. C. Crichton. P. W. Karlsson and A. Pedersen,"Partial Discharges in Ellipsoidal and Spheroidal Voids", IEEE Trans. Elect. Insul., Vol. 24, pp. 335-342, 1989.

[113] I. W. McAllister,"Partial Discharges in Spheroidal Voids: Void Orientation", IEEE Trans. Diel. Elect. Insul., Vol. 4, pp. 456-461, 1997.

[114] 1. W. McAllister and G. C. Crichton, "Influence of Bulk Dielectric Polarization upon Partial Discharge Transients: Effect of Heterogeneous Dielectric Geometry", IEEE Trans. Diel. Elect. Insul., Vol. 7, pp. 124-132, 2000.

[115] H. Edin, “The Influence of Dielectric Polarisation on the Measured PD Charge in a Two-Layer Dielectric System", Nordic Insulation Symposium (NORD-IS 99), Copenhagen, pp. 433-441, 1999. 
[116] I. W. McAllister and G. C. Crichton, "Influence of Bulk Dielectric Polarization upon PD Transients: Effect of Multiple Dielectric Layers", Eleventh International Symposium on High Voltage Engineering, London 1999. IEE Conference Publication No.467, Vol. 4, pp. 71-74, 1999.

[117] I. W. McAllister and G. C. Crichton,"Influence of Void Geometry and Bulk Di-electric Polarization upon PD Transients", in L. G. Christophorou and J. K. Olthoff (eds.), Gaseous Dielectrics VIII, Kluwer Academic/Plenum Publishers, New York, pp. 283-288, 1998.

[118] I. W. McAllister and G. C. Crichton,"Influence of Bulk Dielectric Polarization and Void Geometry upon PD Transients", 1999 Annual Report, Conference on Electrical Insulation and Dielectric Phenomena, Austin TX. IEEE Publication 99CH36319, pp. 197-201, 1999.

[119] N. Achatz, A. Diessner and E. Kynast,"X-Ray Induced Partial Discharges in Smal Hollow Glass Spheres and in GIS Insulators", 9th International Symposium on High Voltage Engineering, Graz, Paper 5599, 1995.

[120] R. Kurrer, K. Klunzinger, K. Feser, N. de Kock and D. Sologuren, "Sensitivity of the UHF-Method for Defects in GIS with Regard to On-line Partial Discharge Detection", Conference Record of the 1996 IEEE International Symposium on Electrical Insulation, Montreal. IEEE Publication 96CH3597-2, pp. 95-98, 1996.

[121] I. W. McAllister, "Influence of Dielectric Polarization upon PD Transients: Use of Hollow Dielectric Spheres", 10th International Symposium on High Voltage Engineering, Montreal, Vol, 4, pp. 427-430, 1997.

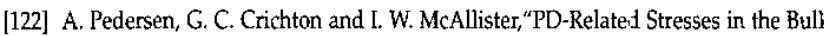
Dielectric and Their Evaluation", 1993 Annual Report, Conference on Electrical Insulation and Dielectric Phenomena, Pocono Manor, PA. IEEE Publication 93CH32698, pp. 474-480, 1993.

[123] I. W. McAllister and T. Johansson, "Partial Discharges and Bulk Dielectric Field Enhancement", 2000 Annual Report, Conference on Electrical Insulation and Dielectric Phenomena, Victoria BC. IEEE Publication 00CH37132, pp. 457-460, 2000.

[124] A. Pedersen, G. C. Crichton and I. W. McAllister, "PD Related Field Enhancement in the Bulk Medium", in L. G. Christophorou and D. R. James (eds.), Gaseous Dielectrics VII, Plenum Press, New York, pp. 223-230, 1994.

[125] A. Pedersen, G. C. Crichton and I. W. McAllister, ${ }^{\prime P D-R e l a t e d ~ S t r e s s e s ~ i n ~ t h e ~ B u l k ~}$ Dielectric for Ellipsoidal Voids", 1994 Annual Report, Confezence on Electrical Insulation and Dielectric Phenomena, Arlington, TX. IEEE Publication 94CH3456-1, pp. 79-84, 1994.

[126] I. W. McAllister,"Decay of Charge Deposited on the Wall of a Gaseous Void", IEEE Trans. Elect. Insul., Vol. 27, pp. 1202-1207, 1992.

In part this paper is based on a presentation given at the Eighth International Symposium on Gaseous Dielectrics, Virginia Beach, VA, 1998

Manuscript was received on 4 February 2002, in revised form 12 March 2002. 